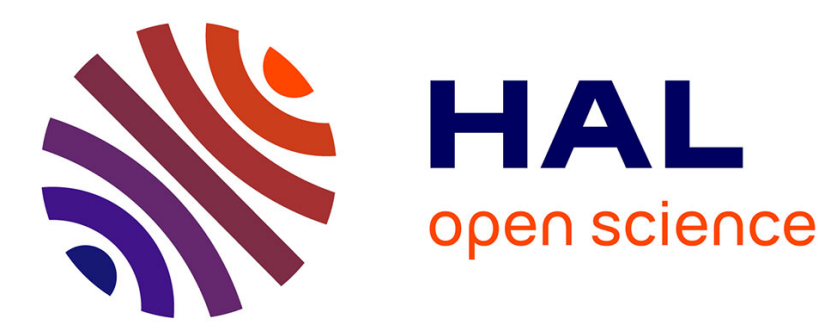

\title{
Locating-Domination and Identification
}

Antoine Lobstein, Olivier Hudry, Irène Charon

\section{To cite this version:}

Antoine Lobstein, Olivier Hudry, Irène Charon. Locating-Domination and Identification. T. Haynes,

S. Hedetniemi \& M. Henning. Topics in Domination in Graphs, pp.251-299, 2020. hal-02916929

\section{HAL Id: hal-02916929 \\ https://hal.science/hal-02916929}

Submitted on 7 Jul 2021

HAL is a multi-disciplinary open access archive for the deposit and dissemination of scientific research documents, whether they are published or not. The documents may come from teaching and research institutions in France or abroad, or from public or private research centers.
L'archive ouverte pluridisciplinaire HAL, est destinée au dépôt et à la diffusion de documents scientifiques de niveau recherche, publiés ou non, émanant des établissements d'enseignement et de recherche français ou étrangers, des laboratoires publics ou privés. 


\title{
Chapter 6 \\ Locating-Domination and Identification
}

Antoine Lobstein, Olivier Hudry, and Irène Charon

\begin{abstract}
Locating-domination and identification are two particular, related, types of domination: a set $C$ of vertices in a graph $G=(V, E)$ is a locating-dominating code if it is dominating and any two vertices of $V \backslash C$ are dominated by distinct sets of codewords; $C$ is an identifying code if it is dominating and any two vertices of $V$ are dominated by distinct sets of codewords. This chapter presents a survey of the major results on locating-domination and on identification.
\end{abstract}

\subsection{Introduction}

Locating-dominating codes were introduced by Slater in 1983 [168], but for more easily accessible sources, see Rall \& Slater [155], or Colbourn, Slater \& Stewart [60]. The term "identifying code" is used in the 1998 paper [134] by Karpovsky, Chakrabarty \& Levitin, which certainly marks the starting point for the blossoming of works on this topic, but the concept is already contained in [158] (Rao, 1993). For both locating-dominating and identifying codes, see the ongoing bibliography at $[142]$.

The graphs $G=(V, E)$ that we shall consider will usually be finite, undirected and connected. Before we proceed, and since we consider domination at distance $r$, we extend the notion of neighborhood: for any integer $r \geqslant 1$, the open $r$-neighborhood of a vertex $v \in V$ is the set $N_{r}(v)=\{u: 0<d(u, v) \leqslant r\}$. The set $N_{r}[v]=N_{r}(v) \cup\{v\}$ is called the closed r-neighborhood of $v$. A code is simply a set of vertices, whose elements are called codewords.

Formally, an $r$-locating-dominating code $C \subseteq V$, abbreviated $r$-LD code, is a distance- $r$ dominating code such that:

\footnotetext{
Antoine Lobstein

Centre National de la Recherche Scientifique, Laboratoire de Recherche en Informatique, e-mail: lobstein@lri.fr

Olivier Hudry

Télécom Paris, Institut polytechnique de Paris, e-mail: hudry@enst.fr

Irène Charon

Télécom Paris, Institut polytechnique de Paris, e-mail: irene.charon@gmail.com
} 


$$
\forall v_{1} \in V \backslash C, \forall v_{2} \in V \backslash C, v_{1} \neq v_{2}: N_{r}\left(v_{1}\right) \cap C \neq N_{r}\left(v_{2}\right) \cap C .
$$

An $r$-locating-dominating code always exists (e.g., $C=V$, or $|C|=|V|-1>0$ whenever $|E|>0$ ). An $r$-identifying code $C \subseteq V$, abbreviated $r$-Id code, is a distance- $r$ dominating code such that:

$$
\forall v_{1} \in V, \forall v_{2} \in V, v_{1} \neq v_{2}: N_{r}\left[v_{1}\right] \cap C \neq N_{r}\left[v_{2}\right] \cap C .
$$

Identifying codes are sometimes called differentiating, mostly when $r=1$. We can see that it is possible to retrieve a vertex $v$ simply by knowing which codewords $r$-dominate $v$. Thus, if each codeword sends an alarm to a central controller whenever there is a malfunctioning vertex in its closed neighborhood, then, only knowing which codewords gave the alarm, the controller can unambiguously retrieve that vertex (if there is at most one). The same is true for a non-codeword in the case of $r$-LD codes. See also [149] for an illustration with smoke detectors.

Two distinct vertices $v_{1} \in V, v_{2} \in V$, are said to be $r$-twins if $N_{r}\left[v_{1}\right]=N_{r}\left[v_{2}\right]$. It is easy to see that a graph $G$ admits an $r$-identifying code if and only if it has no $r$-twins, i.e.,

$$
\forall v_{1} \in V, \forall v_{2} \in V, v_{1} \neq v_{2}: N_{r}\left[v_{1}\right] \neq N_{r}\left[v_{2}\right]
$$

A graph satisfying (6.1) is usually called $r$-identifiable, $r$-twin-free or $r$-distinguishable, or also pointdistinguishing ([175], for $r=1$ ). If three vertices $x, y, z$ are such that $z \in N_{r}[x]$ and $z \notin N_{r}[y]$, we say that $z$-separates $x$ and $y$ in $G$ (note that $z=x$ is possible). A set of vertices $r$-separates $x$ and $y$ if at least one of its elements does. So we can rephrase the definitions: an $r$-LD code (respectively, an $r$-Id code) $r$-dominates every vertex and $r$-separates every pair of non-codewords (respectively, of vertices).

Usually, one is interested in finding the smallest possible size of a code, LD or Id, in a given graph. Such codes are called optimal. Several notations exist; in this chapter, we denote by $L D_{r}(G)$ (respectively, $\left.I d_{r}(G)\right)$ the smallest possible cardinality of an $r$-locating-dominating code (respectively, an $r$-identifying code when $G$ is $r$-twin-free), and we call these numbers the $r$-locating-domination number (respectively, the $r$-identification number) of $G$. They are abbreviated as $r$-LD and $r$-Id numbers, respectively. We may drop $r$ when $r=1$ or when it is irrelevant.

\section{Example}

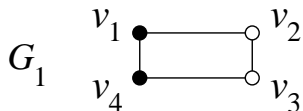

(a)

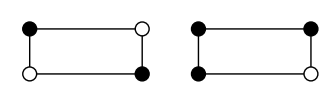

(b) (c)

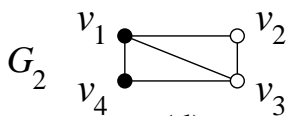

(d)

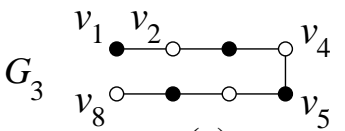

(e)

Fig. 6.1 Different graphs and codes. Black vertices represent codewords.

$r=1$ : (a) $\left\{v_{1}, v_{4}\right\}$ is locating-dominating, not identifying. (b) $\left\{v_{1}, v_{3}\right\}$ is neither locatingdominating nor identifying. (c) $\left\{v_{1}, v_{2}, v_{4}\right\}$ is both locating-dominating and identifying. We have: $L D\left(G_{1}\right)=2, I d\left(G_{1}\right)=3$. (d) $v_{1}$ and $v_{3}$ are twins; $\left\{v_{1}, v_{4}\right\}$ is locating-dominating, and $L D\left(G_{2}\right)=2$. $r=4$ : (e) $v_{4}$ and $v_{5}$ are 4-twins; $\left\{v_{1}, v_{3}, v_{5}, v_{7}\right\}$ is 4-locating-dominating, and $L D_{4}\left(G_{3}\right)=4$. 
The decision problems classically associated with the search of optimal $r$-LD and $r$-Id codes are NP-complete for all $r \geqslant 1$, see Sect. 6.8 on complexity. The following inequalities hold for any $r \geqslant 1$ :

$$
\gamma_{r}(G) \leqslant L D_{r}(G) \leqslant I d_{r}(G) .
$$

Almost all of this chapter is devoted to undirected graphs, but the above definitions can be extended to digraphs, by considering, e.g., insets instead of neighborhoods; see [39], [88], or [59] for an illustration.

The following three theorems are probably as old as the definitions of $r$-dominating, $r$-locatingdominating or $r$-identifying codes.

Theorem 1 Let $r \geqslant 2$ be any integer and $G$ be any graph. A code is 1-dominating (respectively, 1-locating-dominating, 1-identifying) in $G^{r}$, the $r$-th power of $G$, if and only if it is $r$-dominating (respectively, $r$-locating-dominating, $r$-identifying) in $G$.

Theorem 2 Let $r \geqslant 1$ be any integer. Any connected $r$-twin-free graph has order $n=1$ or $n \geqslant 2 r+1$. The only connected $r$-twin-free graph with order $n=2 r+1$ is the path $P_{2 r+1}$.

Any cycle with order $n \geqslant 2 r+2$ is $r$-twin-free.

Theorem 3 Let $r \geqslant 1$ be any integer. Every $r$-dominating code is $(r+1)$-dominating. If $C$ is $r$-dominating (respectively, $r$-locating-dominating, $r$-identifying), then so is any superset $C^{*} \supseteq C$.

\subsection{Possible Values for LD and Id Numbers}

Almost everyone's first results on LD and Id numbers are the following two theorems, which give three easy bounds, whereas Th. 6 is more difficult.

Theorem 4 (a) For any integer $r \geqslant 1$ and any graph $G=(V, E)$ of order $n$, we have

$$
L D_{r}(G) \geqslant\left\lceil\log _{2}\left(n-L D_{r}(G)+1\right)\right\rceil .
$$

(b) For any integer $r \geqslant 1$ and any $r$-twin-free graph $G=(V, E)$ of order $n$, we have

$$
I d_{r}(G) \geqslant\left\lceil\log _{2}(n+1)\right\rceil .
$$

Proof. (a) Let $C$ be any $r$-LD code in $G$. All the $n-|C|$ non-codewords $v \in V \backslash C$ must be given nonempty and distinct sets $N_{r}(v) \cap C$, constructed with the $|C|$ codewords, so $2^{|C|}-1 \geqslant n-|C|$, from which (6.3) follows when $C$ is optimal; (b) the argument is the same, but we have to consider all the $n$ vertices $v \in V$, so $2^{|C|}-1 \geqslant n$.

Theorem 5 For any $r \geqslant 1$ and connected graph $G$ of order $n \geqslant 2$, we have $L D_{r}(G) \leqslant n-1$.

Theorem 6 ([15]) For any $r \geqslant 1$ and connected $r$-twin-free graph $G$ of order $n \neq 1$, we have $I d_{r}(G) \leqslant n-1$.

Proof. We give the short elegant proof by Gravier \& Moncel [98] in 2007, six years after the first proof in [15]. Using Th. 1, we can see that it is sufficient to prove the case $r=1$. We assume that $G=(V, E)$ is a connected 1-twin-free graph, of order $n \geqslant 3$. Let $C_{1}=V \backslash\{a\}$ for $a \in V$ with 
maximum degree in $G$. If $C_{1}$ is identifying, we are done, so we assume that it is not. Because $G$ is connected, $C_{1}$ is a dominating code. If $u$ and $v$ belong to $N[a]$ and because $N[u] \neq N[v]$, we have $N[u] \backslash\{a\} \neq N[v] \backslash\{a\}$, so $C_{1}$ separates $u$ and $v$, and the same is true for $u \notin N[a]$ and $v \notin N[a]$. Therefore, there must be $u \in N[a]$ and $v \notin N[a]$ which are not separated by $C_{1}$, i.e., $N[u] \cap C_{1}=N[v] \cap C_{1}$, or $N[u] \backslash\{a\}=N[v]$.

We claim that $C_{2}=V \backslash\{v\}$ is an identifying code. By the previous discussion with $a$, which did not use the maximum degree assumption yet, it is sufficient to check that every vertex in $N[v]$ is separated from every vertex not in $N[v]$. And indeed: (i) since $N[u]=N[v] \cup\{a\}$, each vertex in $V \backslash(N[v] \cup\{a\})$ is separated from each vertex in $N[v]$ by $u$; (ii) the vertex $a$ is separated from $v$ by itself; (iii) the vertex $a$ is separated from each vertex $a^{\prime} \in N[v] \backslash\{v\}$, for otherwise, $N\left[a^{\prime}\right]=N[a] \cup\{v\}$, i.e., $a^{\prime}$ has degree greater than $a$, a contradiction.

The previous result actually holds for all graphs, finite or infinite, with bounded degree. There exist (infinite) graphs (with unbounded degree) such that the only 1-identifying code is the whole vertex set [15]: take two copies, $G_{1}$ and $G_{2}$, of the infinite complete graph with vertex set $\mathbb{Z}$, and link $i \in V\left(G_{1}\right)$ to $j \in V\left(G_{2}\right)$ if and only if $i \geqslant j$.

\subsubsection{Reaching the Bounds}

Graphs exist that meet the lower and upper bounds given previously. Theorem $9(\mathrm{a})$ even characterizes the graphs $G$ of order $n$ such that $L D(G)=n-1$, as will Th. 12 for 1-identifying codes.

Theorem 7 ([43]) Let $r \geqslant 1$ and $n$ be integers such that $n \geqslant 2^{2 r+1}+2 r+1$. There exists a connected graph of order $n$ admitting an r-locating-dominating code achieving the lower bound (6.3).

Theorem 8 ([134, for $r=1]),([64]),([43])$ Let $r \geqslant 1$ and $n$ be integers such that $n \geqslant 2^{2 r}$. There exists a connected graph of order $n$ admitting an $r$-identifying code of size $\left\lceil\log _{2}(n+1)\right\rceil$.

For $r=1$, Th. 8 holds for all $n \geqslant 3$; for $r=2$, it holds if and only if $n \geqslant 6$.

Theorem 9 (a) ([169]), ([170]) A connected graph $G$ of order $n \geqslant 2$ is such that $L D(G)=n-1$ if and only if $G$ is the star $K_{1, n-1}$ or the complete graph $K_{n}$.

(b) For all $r \geqslant 1$ and $n \geqslant 2$, we have $L D_{r}\left(K_{n}\right)=n-1$.

Theorem 10 For all $n \geqslant 3$, there exists a connected graph $G$ of order $n$ such that $I d(G)$ achieves the upper bound $n-1$.

An easy example of such a graph is the star, see Fig. 6.2(a). We give two more examples, which will be used for the construction of graphs $G$ such that $I d_{r}(G)=n-1$ for all $r$.

In the following, a vertex is $r$-universal if it $r$-dominates all the vertices in the graph. Let $p \geqslant 2$.

(a) Take $G_{2 p}^{*}=\left(V_{2 p}^{*}, E_{2 p}^{*}\right)$, with $V_{2 p}^{*}=\left\{v_{0}, v_{1}, \ldots, v_{2 p-1}\right\}, E_{2 p}^{*}=\left\{v_{i} v_{j}: v_{i} \in V_{2 p}^{*}, v_{j} \in V_{2 p}^{*}, i \neq\right.$ $j, i \neq j+p \bmod 2 p\}$ : the graph $G_{2 p}^{*}$ has even order and is the complete graph $K_{2 p}$ minus the edges of a perfect matching.

(b) Take $G_{2 p+1}^{*}$, obtained from $G_{2 p}^{*}$ simply by adding one 1-universal vertex; its order is odd. In view of Sect. 6.3, the following theorem gives the domination, locating-domination and identification numbers of these two classes of graphs, as well as for the star. 
(a)

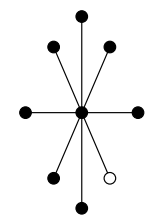

(b)

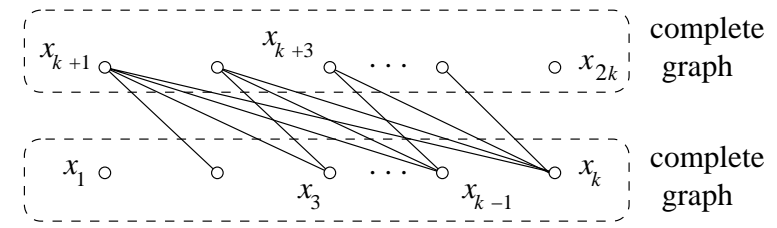

Fig. 6.2 (a) The star $K_{1,8}$, with an optimal identifying code (of size 8), which is also an optimal locatingdominating code. (b) The graph $A_{k}$.

Theorem 11 Let $p \geqslant 2$ and $n \geqslant 3$. Then

(a) $\gamma\left(G_{2 p}^{*}\right)=2, \operatorname{LD}\left(G_{2 p}^{*}\right)=p$ and $\operatorname{Id}\left(G_{2 p}^{*}\right)=2 p-1$;

(b) $\gamma\left(G_{2 p+1}^{*}\right)=1, L D\left(G_{2 p+1}^{*}\right)=p$ and $\operatorname{Id}\left(G_{2 p+1}^{*}\right)=2 p$;

(c) $\gamma\left(K_{1, n-1}\right)=1, \operatorname{LD}\left(K_{1, n-1}\right)=n-1$ and $\operatorname{Id}\left(K_{1, n-1}\right)=n-1$.

It is an interesting fact, published in 2010 [82], that it is possible to characterize the graphs $G$ with $\operatorname{Id}(G)=n-1$. For two graphs $G=(V, E)$ and $G^{\prime}=\left(V^{\prime}, E^{\prime}\right)$, let $G \bowtie G^{\prime}=\left(V \cup V^{\prime}, E \cup\right.$ $\left.E^{\prime} \cup\left\{v v^{\prime}: v \in V, v^{\prime} \in V^{\prime}\right\}\right)$ be their join graph. Let $A_{k}=\left(V_{k}, E_{k}\right)$, with $V_{k}=\left\{x_{1}, \ldots, x_{2 k}\right\}$ and $E_{k}=\left\{x_{i} x_{j}:|i-j| \leqslant k-1\right\}$, see Fig. 6.2(b). Note that $A_{1}$ consists of two isolated vertices, and for $k \geqslant 2, A_{k}$ is the $(k-1)$-th power of the path $P_{2 k}$. Finally, let $\mathcal{A}$ be the closure of all the graphs $A_{i}$ for $\bowtie$.

Theorem 12 (Foucaud et al. [82]), (Foucaud et al. [83]) A connected graph $G$ of order $n \geqslant 3$ is such that $\operatorname{Id}(G)=n-1$ if and only if $G$ is the star or belongs to the set of graphs $\mathcal{A} \cup\left\{A \bowtie K_{1}: A \in \mathcal{A}\right\}$.

Note that $G_{4}^{*} \simeq A_{1} \bowtie A_{1}, G_{6}^{*} \simeq G_{4}^{*} \bowtie A_{1}, \ldots, G_{2 p}^{*} \simeq G_{2 p-2}^{*} \bowtie A_{1}$, and that $G_{2 p+1}^{*} \simeq G_{2 p}^{*} \bowtie K_{1}$. We now turn to the case $r \geqslant 2$ : are there graphs $G$ such that $I d_{r}(G)=n-1$ ? (as we have already seen, complete graphs give a trivial positive answer for $r$-LD codes). A crucial fact is that the two graphs $G_{2 p}^{*}$ and $G_{2 p+1}^{*}$ admit $r$-th roots for any $r$, if $p$ is sufficiently large. More precisely:

Theorem 13 ([43]) Let $r \geqslant 2$ and $p \geqslant 2$ be integers.

(a) If $2 p \geqslant 3 r^{2}$, then there exists a graph $G_{2 p}$ of order $2 p$ such that $\left(G_{2 p}\right)^{r}=G_{2 p}^{*}$.

(b) If $2 p \geqslant 3 r^{2}$, then there exists a graph $G_{2 p+1}$ of order $2 p+1$ such that $\left(G_{2 p+1}\right)^{r}=G_{2 p+1}^{*}$.

Corollary 1 (a) For $n \geqslant 3 r^{2}$, there exists a graph $G_{n}$ of even order $n$ such that $\gamma_{r}\left(G_{n}\right)=2$, $L D_{r}\left(G_{n}\right)=\frac{n}{2}$ and $I d_{r}\left(G_{n}\right)=n-1$.

(b) For $n \geqslant 3 r^{2}+1$, there exists a graph $G_{n}$ of odd order $n$ such that $\gamma_{r}\left(G_{n}\right)=1, L D_{r}\left(G_{n}\right)=\frac{n-1}{2}$ and $I d_{r}\left(G_{n}\right)=n-1$.

Proof. Use Ths. 11(a)-(b), 13 and 1.

\subsubsection{Reaching All Intermediate Values}

As for the lower and upper bounds, constructions show how to achieve all intermediate values.

Theorem 14 ([42]) Let $r \geqslant 1$ and $c \geqslant 5 r^{2}+5 r+1$. For $n$ between $c+1$ and $2^{c}+c-1$, there exists a connected graph $G$ of order $n$ such that $L D_{r}(G)=c$. 
For Id codes, the case $r=1$ is easy and one can even address the special case of bipartite graphs.

Theorem 15 ([41]) For $n \geqslant 3$ and for any integer $c$ between $\left\lceil\log _{2}(n+1)\right\rceil$ and $n-1$, there exists a connected bipartite graph $G$ with $n$ vertices such that $\operatorname{Id}(G)=c$.

Theorem $16([41])$ Let $r \geqslant 1$ and $c \geqslant 5 r^{2}+5 r+1$. For $n$ between $c+1$ and $2^{c}-1$, there exists a connected graph $G$ of order $n$ such that $I d_{r}(G)=c$.

\subsection{The Cost of Locating-Domination and Identification}

The inequalities $\gamma_{r}(G) \leqslant L D_{r}(G) \leqslant I d_{r}(G)$ in (6.2) express that locating-domination is more "expensive" than domination, and identification is more expensive than locating-domination. In this section, we compare the respective "costs" for these three notions. More precisely, denoting, for graphs of order $n$,

$\mathcal{G}_{r, n}=\{G: r$-twin-free, connected, $n \geqslant 2 r+1\}$ and $\mathcal{G}_{r, n}^{t w}=\{G:$ with $r$-twins, connected, $n \geqslant 2\}$,

we study the following maximum and minimum differences:

- $F_{I d, L D}(r, n)=\max _{G \in \mathcal{G}_{r, n}}\left\{I d_{r}(G)-L D_{r}(G)\right\}, f_{I d, L D}(r, n)=\min _{G \in \mathcal{G}_{r, n}}\left\{I d_{r}(G)-L D_{r}(G)\right\}$,

- $F_{I d, \gamma}(r, n)=\max _{G \in \mathcal{G}_{r, n}}\left\{I d_{r}(G)-\gamma_{r}(G)\right\}, f_{I d, \gamma}(r, n)=\min _{G \in \mathcal{G}_{r, n}}\left\{I d_{r}(G)-\gamma_{r}(G)\right\}$.

In order to see the influence of the twin-free property on locating-domination and domination, we distinguish, for dominating and locating-dominating codes, between two cases, and study the following functions:

- $F_{L D, \gamma}(r, n)=\max _{G \in \mathcal{G}_{r, n}}\left\{L D_{r}(G)-\gamma_{r}(G)\right\}, f_{L D, \gamma}(r, n)=\min _{G \in \mathcal{G}_{r, n}}\left\{L D_{r}(G)-\gamma_{r}(G)\right\}$;

these two functions are considered on the same set of graphs (the twin-free graphs) as the four functions involving identification, unlike the two functions below:

- $F_{L D, \gamma}^{t w}(r, n)=\max _{G \in \mathcal{G}_{r, n}^{t w}}\left\{L D_{r}(G)-\gamma_{r}(G)\right\}, f_{L D, \gamma}^{t w}(r, n)=\min _{G \in \mathcal{G}_{r, n}^{t w}}\left\{L D_{r}(G)-\gamma_{r}(G)\right\}$.

Finally, if we want to consider all the connected graphs of order $n$, twin-free or not, the result is obviously obtained by taking $\max \left\{F_{L D, \gamma}(r, n), F_{L D, \gamma}^{t w}(r, n)\right\}$ and $\min \left\{f_{L D, \gamma}(r, n), f_{L D, \gamma}^{t w}(r, n)\right\}$.

Note that [20] characterizes the trees $T$ such that $L D_{1}(T)=\gamma_{1}(T)$ or $I d_{1}(T)=\gamma_{1}(T)$. Most results in this section on cost (namely, Ths. 19-26) are due to Hudry \& Lobstein [124] in 2020.

\subsubsection{Preliminary Results}

Theorem 17 ([147]), ([102, p. 41]) If $G$ has order $n$ and no isolated vertices, then $\gamma(G) \leqslant \frac{n}{2}$.

Theorem 18 ([96]) If $G$ is 1-twin-free, then $\operatorname{Id}(G) \leqslant 2 L D(G)$.

Thanks to Th. 1 on the powers of graphs, the previous two results are true also for $\gamma_{r}(G), I_{r}(G)$ (for $r$-twin-free graphs) and $L D_{r}(G)$, for any $r \geqslant 2$. 


\subsubsection{Identification vs Domination}

First, we construct an infinite family of graphs $G_{n}^{*}$ of order $n$ satisfying $I d_{r}\left(G_{n}^{*}\right)=\gamma_{r}\left(G_{n}^{*}\right)$. These graphs have order $n=k(r+1), k \geqslant 2 r+2$, and are informally given by Fig. $6.3(\mathrm{a})$.
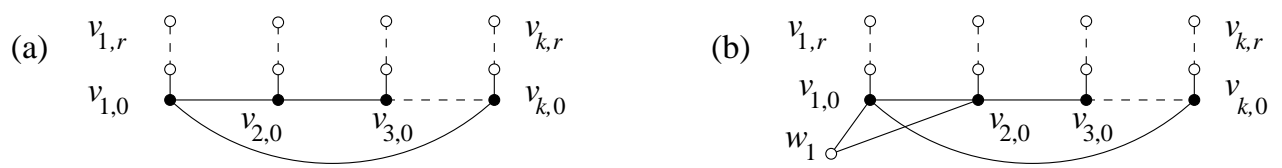

Fig. 6.3 The $k$ black vertices represent codewords. (a) The graph $G_{n}^{*}$. (b) The graph $G_{n+1}$.

Theorem 19 (a) For all $r \geqslant 1, k \geqslant 2 r+2$ and $n=k(r+1)$, we have $\gamma_{r}\left(G_{n}^{*}\right)=I d_{r}\left(G_{n}^{*}\right)=k$.

(b) For all $r \geqslant 1$ and $n \geqslant(2 r+2)(r+1)$, we have $f_{I d, \gamma}(r, n)=0$, and obviously $f_{I d, L D}(r, n)=$ $f_{L D, \gamma}(r, n)=0$.

Proof. (a) The $k$ vertices $v_{i, r}$ must be $r$-dominated by at least one codeword, and no vertex can $r$-dominate two such vertices, so $\gamma_{r}\left(G_{n}^{*}\right) \geqslant k$. On the other hand, the code $C=V_{0}$ represented by the black vertices on Fig. 6.3(a) has cardinality $k$, and it is straightforward to check that it is $r$-identifying. Note in particular that vertices in $\left\{v_{1,0}, v_{2,0}, \ldots, v_{k, 0}\right\}$ are $r$-dominated by exactly $2 r+1$ codewords (this is where the assumption $k \geqslant 2 r+2$ is crucial), and more generally, vertices $v_{i, j} \in\left\{v_{i, 1}, v_{i, 2}, \ldots, v_{i, r}\right\}$ are $r$-dominated by an odd number, namely $2 r-2 j+1$, of codewords, for all $i \in\{1,2, \ldots, k\}$ and $j \in\{1,2, \ldots, r\}$. So $k \leqslant \gamma_{r}\left(G_{n}^{*}\right) \leqslant I d_{r}\left(G_{n}^{*}\right) \leqslant k$.

(b) Then it is easy to see that we can reach all intermediate values between $k(r+1)$ and $(k+1)(r+1)-1$. Figure $6.3(\mathrm{~b})$ illustrates the case $k(r+1)+1$, with one additional vertex, $w_{1}$, which is $r$-dominated by an even number of codewords in $G_{n+1}$.

For $r=1$, Th. 19 starts at $n=8$, for $k=4$, but we can fill in the holes: $f_{I d, \gamma}(1, n)=1$ for $n \in\{3,4,5\}$, and for $n \in\{6,7\}, f_{I d, \gamma}(1, n)=0=f_{I d, L D}(1, n)=f_{L D, \gamma}(1, n)$.

Now how large can the difference $I d_{r}(G)-\gamma_{r}(G)$ be? We know that it is at most $n-2$, obtained by graphs $G$ with $I d_{r}(G)=n-1$ and $\gamma_{r}(G)=1$.

Theorem 20 (a) For all $n \geqslant 3$, we have $F_{I d, \gamma}(1, n)=n-2$.

(b) For all $r \geqslant 2$ and even $n \geqslant 3 r^{2}$, we have $F_{I d, \gamma}(r, n)=n-3$.

(c) For all $r \geqslant 2$ and odd $n \geqslant 3 r^{2}+1$, we have $F_{I d, \gamma}(r, n)=n-2$.

Proof. Use the star for (a), Cor. 1(b) for (c). For (b), observe that, among all the graphs $G$ of even order $n$ such that $\operatorname{Id}(G)=n-1$ (see Th. 12), none of them, except the star, contains a 1-universal vertex, i.e., is such that $\gamma(G)=1$. But the star cannot be the power of any graph. Therefore, for $r \geqslant 2$, there can exist no graph $G$ with even order $n$ such that $I d_{r}(G)=n-1$ and $\gamma_{r}(G)=1$, since the $r$-th power of this graph would contradict our previous observation; consequently the difference $I d_{r}(G)-\gamma_{r}(G)$ is at most $n-3$. On the other hand, Cor. 1(a) gives an example achieving $n-3$.

\subsubsection{Identification vs Locating-Domination}

Theorem 21 (a) (= Th. 19(b)) For all $r \geqslant 1$ and $n \geqslant(2 r+2)(r+1)$, we have $f_{I d, L D}(r, n)=0$. 
(b) For all $n \geqslant 3$, we have $f_{I d, L D}(1, n)=0$.

Theorem 22 (a) For all $r \geqslant 1$ and $n \geqslant 3 r^{2}+1$, we have $F_{I d, L D}(r, n)=\left\lceil\frac{n}{2}\right\rceil-1$.

(b) We have $F_{I d, L D}(1,3)=0$.

Proof. We know (cf. Th. 18) that any (connected) $r$-twin-free graph $G$ is such that $I d_{r}(G) \leqslant$ $2 L D_{r}(G)$. Therefore, $I d_{r}(G)-L D_{r}(G) \leqslant I d_{r}(G)-\frac{I d_{r}(G)}{2} \leqslant \frac{n-1}{2}$, leading to $I d_{r}(G)-L D_{r}(G) \leqslant$ $\left\lceil\frac{n}{2}\right\rceil-1$. On the other hand, Cor. $1(\mathrm{a})-(\mathrm{b})$ provides graphs reaching $\left\lceil\frac{n}{2}\right\rceil-1$.

\subsubsection{Domination vs Locating-Domination}

The two cases, without and with twins, show quite a difference between $F_{L D, \gamma}$ and $F_{L D, \gamma}^{t w}$.

\subsubsection{Domination vs Locating-Domination in Twin-Free Graphs}

Using Th. 19(b), the sentence following its proof, and the paths $P_{4}$ and $P_{5}$, we obtain the following.

Theorem 23 (a) For all $n \geqslant 4$, we have $f_{L D, \gamma}(1, n)=0 ; f_{L D, \gamma}(1,3)=1$.

(b) For all $r \geqslant 2$ and $n \geqslant(2 r+2)(r+1)$, we have $f_{L D, \gamma}(r, n)=0$.

Theorem 24 (a) For all $n \geqslant 3$, we have $F_{L D, \gamma}(1, n)=n-2$.

(b) For all $r \geqslant 2$ and $n \geqslant 3 r^{2}+1$, we have $F_{L D, \gamma}(r, n) \geqslant \frac{n}{2}-2$.

(c) For all $r \geqslant 2$ and $n \geqslant 2 r+1$, we have $F_{L D, \gamma}(r, n) \leqslant n-3$.

Proof. (a) The star shows that $F_{L D, \gamma}(1, n)=n-2$. (b) For any $r$, Cor. 1(a)-(b) immediately gives examples proving that $F_{L D, \gamma}(r, n) \geqslant \frac{n}{2}-2$. (c) The characterization of the graphs $G$ of order $n$ such that $I d(G)=n-1$ (Th. 12) gives graphs which, apart from the star which is not the power of any graph, are such that $L D(G) \leqslant n-2$. This allows to conclude that $F_{L D, \gamma}(r, n) \leqslant n-3$, using the powers of graphs as in the proof of Th. 20 .

Improvements are possible on Th. 24(b) when $r=2$.

Theorem 25 (a) Let $n=8 t \geqslant 24$. Then $F_{L D, \gamma}(2, n) \geqslant 5 t-3$.

(b) Let $n=8 t+i \geqslant 24$, with $1 \leqslant i \leqslant 7$. Then $F_{L D, \gamma}(2, n) \geqslant 5 t+i-6$.

\subsubsection{Domination vs Locating-Domination in Graphs with Twins}

Theorem 26 (a) For $r \geqslant 1$ and $n \geqslant 2$, we have $F_{L D, \gamma}^{t w}(r, n)=n-2$.

(b) For $n \in\{2,5\}$ or $n \geqslant 7$, we have $f_{L D, \gamma}^{t w}(1, n)=0$; for $n \in\{3,4,6\}$, we have $f_{L D, \gamma}^{t w}(1, n)=1$.

(c) For all $r \geqslant 2$ and $n \geqslant(2 r+2)(r+1)+1$, we have $f_{L D, \gamma}^{t w}(r, n)=0$.

To conclude this section on compared costs, we can see that for $r=1$, we have exact values for all $n$ and functions $f$ and $F$. For $r \geqslant 2$, most results are exact but valid for $n$ large. One open problem is to establish results for all $n$, another to reduce the gap between lower and upper bounds for $F_{L D, \gamma}(r, n)$, cf. Ths. 24(b)-(c) and 25. Note that all open problems and conjectures mentioned throughout this chapter are gathered at its end. 


\subsection{Specific Families of Graphs}

We survey some well-known and some not so well-known families of graphs.

\subsubsection{Infinite Grids and Strips}

The four infinite 2-dimensional grids $G_{S}$ (square), $G_{T}$ (triangular), $G_{K}$ (king), and $G_{H}$ (hexagonal), partially represented in Fig. 6.4, have been much studied in the literature, specially with respect to identifying codes, and the densities $\partial_{r}^{L D}\left(G_{\pi}\right)$ and $\partial_{r}^{I d}\left(G_{\pi}\right)$ of optimal $r$-LD and $r$-Id codes investigated for $\pi \in\{S, T, K, H\}$. One can also consider the infinite strips $G_{\pi}^{[k]}$ of height $k \geqslant 1$ (the case $k=1$ gives the infinite path, see also below, Sect. 6.4.2). We remind that the density of a code $C$ in $G_{\pi}^{[k]}$ can be defined for every $k \geqslant 1$ by $\partial\left(C, G_{\pi}^{[k]}\right)=\limsup _{n \rightarrow \infty} \frac{\left|C \cap N_{n}[v]\right|}{\left|N_{n}[v]\right|}$, where $v$ is an arbitrary vertex.

(a)

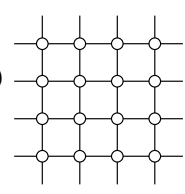

(b)

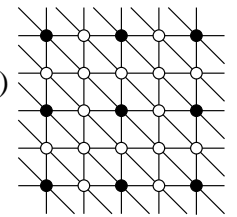

(c)

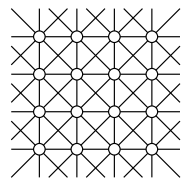

(d)

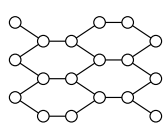

honeycomb

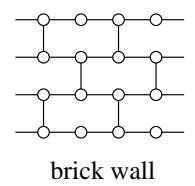

Fig. 6.4 Partial representations of the four grids: (a) the square grid; (b) the triangular grid: black vertices are codewords (cf. Th. 32); (c) the king grid; (d) the hexagonal grid (with two possible representations).

Some constructions of codes are obtained by heuristics searching for small subcodes inside tiles that will be repeated periodically [38], see Fig. 6.5 for a first example.

Fig. 6.5 A periodic 5identifying code in the square grid $G_{S}$, of density $2 / 25$. Codewords are in black.

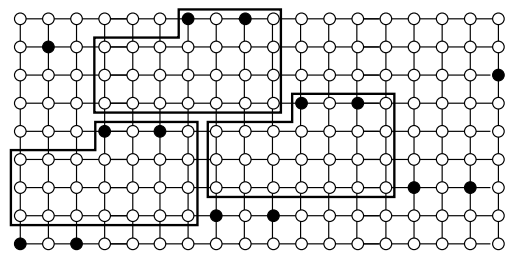

We give, as far as we know, the best lower and upper bounds, for grids and strips. Note how few results there are for $r$-LD codes when $r \neq 1$. 


\subsubsection{The Square Grid}

The square grid, $G_{S}$, has vertex set $V_{S}=\mathbb{Z} \times \mathbb{Z}$ and edge set $E_{S}=\{u v: u-v \in\{(1,0),(0,1)\}$. Figure 6.6 will give constructions proving the upper bounds for Ths. 27(a) and 28, Fig. 6.7 for Ths. 27(c) and 30(b).

\section{- Locating-dominating codes}

Theorem 27 ([171]), ([172]) (a) We have $\partial_{1}^{L D}\left(G_{S}\right)=\frac{3}{10}$.

(b) We have $\partial_{1}^{L D}\left(G_{S}^{[1]}\right)=\frac{2}{5}$.

(c) We have $\partial_{1}^{L D}\left(G_{S}^{[2]}\right)=\frac{3}{8}=0.375, \partial_{1}^{L D}\left(G_{S}^{[3]}\right)=\frac{1}{3}$.

\section{- Identifying codes}

The following upper bound $\partial_{1}^{I d}\left(G_{S}\right) \leqslant \frac{7}{20}$ is from [54] in 1999, the lower bound $\partial_{1}^{I d}\left(G_{S}\right) \geqslant \frac{7}{20}$ by Ben-Haim \& Litsyn [13] in 2005.

Theorem 28 We have $\partial_{1}^{I d}\left(G_{S}\right)=\frac{7}{20}$.

(a)

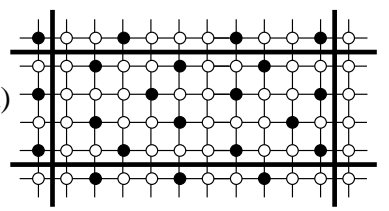

(b)

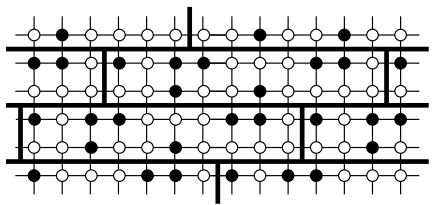

Fig. 6.6 Black vertices are the elements of a periodic code in $G_{S}$, which is (a) 1-LD with density $\frac{3}{10}$, (b) 1-Id with density $\frac{7}{20}$.

The following general lower bounds come from [33], the lower bound in (b) from [128], and all the upper bounds from [117].

Theorem 29 (a) For every $r \geqslant 1$, we have $\frac{3}{8 r+4} \leqslant \partial_{r}^{I d}\left(G_{S}\right) \leqslant\left\{\begin{array}{ll}\frac{2}{5 r} 2 r & : r \text { even } \\ \frac{5 r^{2}-2 r+1}{}: r \text { odd }\end{array}\right.$. When $r$ increases, these bounds are close to $\frac{3}{8 r}=\frac{0.375}{r_{5}}$ and $\frac{2}{5 r}$.

(b) We have $\frac{6}{35} \approx 0.17143 \leqslant \partial_{2}^{I d}\left(\stackrel{8 r}{G}_{S}\right) \leqslant \frac{r_{5}}{29} \approx 0.17241$.

The previous upper bounds have been improved in [38], using heuristics, for $r \in\{3,4,5,6\}$. The strips of all heights have also been studied, for $r=1$; in (d) below, the upper bound is from [22], the lower bound from [126].

Theorem 30 (a) ([94]) We have $\partial_{1}^{I d}\left(G_{S}^{[1]}\right)=\frac{1}{2}$.

(b) ([64]), ([65]) We have $\partial_{1}^{I d}\left(G_{S}^{[2]}\right)=\frac{3}{7} \approx 0.42857$.

(c) ([22]) We have $\partial_{1}^{I d}\left(G_{S}^{[3]}\right)=\frac{7}{18} \approx 0.38889$.

(d) We have $\partial_{1}^{I d}\left(G_{S}^{[4]}\right)=\frac{11}{28} \approx 0.39286$.

(e) $([126])$ We have $\partial_{1}^{I d}\left(G_{S}^{[5]}\right)=\frac{19}{50}=0.38$.

(f) ([23]) For $k \geqslant 6$, we have $\frac{7}{20}+\frac{1}{20 k} \leqslant \partial_{1}^{I d}\left(G_{S}^{[k]}\right) \leqslant \frac{7}{20}+\frac{3}{10 k}$. 
(a)

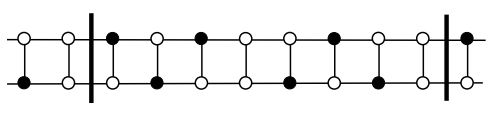

(b)

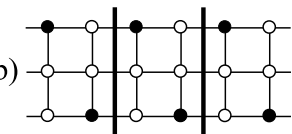

(c)

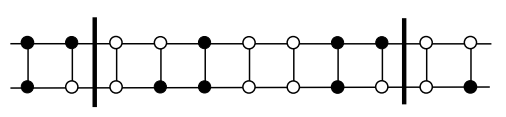

Fig. 6.7 Black vertices are the elements of a periodic code which is (a) 1-LD with density $\frac{3}{8}$ in $G_{S}^{[2]}$, (b) 1-LD with density $\frac{1}{3}$ in $G_{S}^{[3]}$, (c) 1-Id with density $\frac{3}{7}$ in $G_{S}^{[2]}$.

\subsubsection{The Triangular Grid}

The triangular grid, or square grid with one diagonal, $G_{T}$, has vertex set $V_{T}=\mathbb{Z} \times \mathbb{Z}$ and edge set $E_{T}=\{u v: u-v \in\{(1,0),(0,1),(1,-1)\}\}$. Figure 6.8 will give constructions proving the upper bounds in $G_{T}^{[3]}$ for Ths. 31(b) and 34(a).

\section{- Locating-dominating codes}

Theorem 31 (a) ([107]) We have $\partial_{1}^{L D}\left(G_{T}\right)=\frac{13}{57} \approx 0.22807$.

(b) $([22])$ We have $\partial_{1}^{L D}\left(G_{T}^{[2]}\right)=\frac{1}{3}, \partial_{1}^{L D}\left(G_{T}^{[3]}\right)=\frac{3}{10}$.

\section{- Identifying codes}

Theorem 32 (Karpovsky, Chakrabarty \& Levitin [134]) We have $\partial_{1}^{I d}\left(G_{T}\right)=\frac{1}{4}$.

Proof. The upper bound is proved by the construction given in Fig. 6.4(b), which has the property $(\mathrm{P})$ that every codeword is dominated by exactly one codeword (itself) and every non-codeword is dominated by exactly two codewords (either horizontally or vertically or diagonally). This proves that the code is indeed 1-identifying, and moreover that it is best possible.

Property $(\mathrm{P})$ is at the root of Th. 70 when $r=1$. Another example of a graph and a code having this property is the cycle of length $2 p \geqslant 6$, with $p$ pairwise nonadjacent vertices forming the code, see Th. 44. Property (P) cannot be true for $r>1$ [57].

Theorem 33 (a) ([33]) For every $r \geqslant 1$, we have $\frac{2}{6 r+3} \leqslant \partial_{r}^{I d}\left(G_{T}\right) \leqslant\left\{\begin{array}{l}\frac{1}{2 r+2}: r \in\{1,2,3\} \bmod 4 \\ \frac{1}{2 r+4}: r=0 \bmod 4\end{array}\right.$.

When $r$ increases, these bounds are close to $\frac{1}{3 r}$ and $\frac{1}{2 r}$.

(b) $([38])$ We have $\partial_{3}^{I d}\left(G_{T}\right) \leqslant \frac{2}{17} \approx 0.11765$ and $\partial_{5}^{I} d\left(G_{T}\right) \leqslant \frac{1}{13} \approx 0.07692$.

Results on the different triangular strips are available when $r=1$.

Theorem $34([66])$ (a) We have $\partial_{1}^{I d}\left(G_{T}^{[2]}\right)=\frac{1}{2}, \partial_{1}^{I d}\left(G_{T}^{[3]}\right)=\partial_{1}^{I d}\left(G_{T}^{[4]}\right)=\frac{1}{3}, \partial_{1}^{I d}\left(G_{T}^{[5]}\right)=\frac{3}{10}$, and $\partial_{1}^{I d}\left(G_{T}^{[6]}\right)=\frac{1}{3}$.

(b) For odd $k \geqslant 7$, we have $\partial_{1}^{I d}\left(G_{T}^{[k]}\right)=\frac{1}{4}+\frac{1}{4 k}$.

(c) For even $k \geqslant 8$, we have $\frac{1}{4}+\frac{1}{4 k} \leqslant \partial_{1}^{I d}\left(G_{T}^{[k]}\right) \leqslant \frac{1}{4}+\frac{1}{2 k}$.

\subsubsection{The King Grid}

The king grid, or square grid with two diagonals, $G_{K}$, has vertex set $V_{K}=\mathbb{Z} \times \mathbb{Z}$ and edge set $E_{K}=\{u v: u-v \in\{(1,0),(0,1),(1,-1),(1,1)\}\}$. Its name comes from the fact that, on an infinite 
(a)

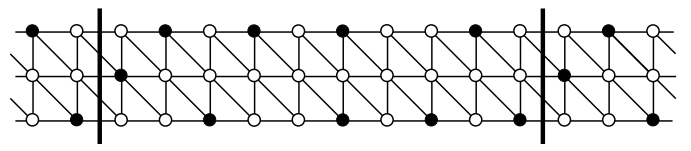

(b)

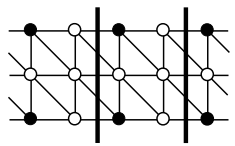

Fig. 6.8 Black vertices are the elements of a periodic code in $G_{T}^{[3]}$ which is (a) 1-LD with density $\frac{3}{10}$, (b) 1-Id with density $\frac{1}{3}$.

chessboard, the $r$-neighborhood of a vertex $v$ is the set of vertices that a king, starting from $v$, can reach in at most $r$ moves. Figure 6.9 will give constructions proving the upper bounds for Ths. 35,37 and $38(\mathrm{~b})$.

\section{- Locating-dominating codes}

Theorem 35 ([113]) We have $\partial_{1}^{L D}\left(G_{K}\right)=\frac{1}{5}$.

For $r>1$, there are good bounds on $\partial_{r}^{L D}\left(G_{K}\right)$, and the first two strips are solved for $r=1$.

Theorem $36([152])$ For every $r \geqslant 1$, we have $\frac{1}{4 r+2} \leqslant \partial_{r}^{L D}\left(G_{K}\right) \leqslant\left\{\begin{array}{ll}\frac{1}{4 r} r_{r+1}: r \text { even } \\ \frac{4 r(r+1)+2}{} \text { odd }\end{array}\right.$. When $r$ increases, these bounds are all equivalent to $\frac{1}{4 r}$.

Theorem $37([22])$ We have $\partial_{1}^{L D}\left(G_{K}^{[2]}\right)=\frac{1}{2}, \partial_{1}^{L D}\left(G_{K}^{[3]}\right)=\frac{4}{15} \approx 0.26667$.

- Identifying codes

It is remarkable that the best density is known for all $r \geqslant 1$ for identification [34] (2004). In the following theorem, the lower bound on $\partial_{1}^{I d}\left(G_{K}\right)$ comes from [58], the upper bound from [38].

Theorem 38 (a) (Charon et al. [34]) For every $r>1$, we have $\partial_{r}^{I d}\left(G_{K}\right)=\frac{1}{4 r}$.

(b) We have $\partial_{1}^{I d}\left(G_{K}\right)=\frac{2}{9} \approx 0.22222$.

(a)

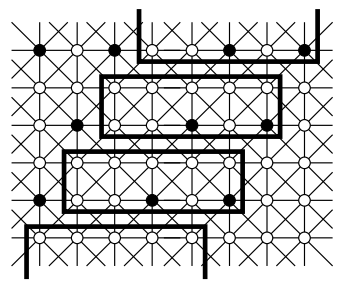

(b)

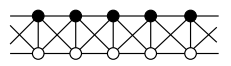

(c)

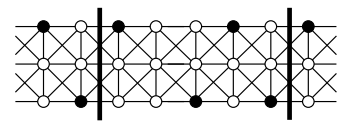

(d)

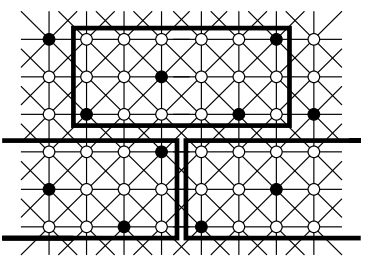

Fig. 6.9 Black vertices are the elements of a periodic code which is (a) 1-LD with density $\frac{1}{5}$ in $G_{K}$, (b) 1-LD with density $\frac{1}{2}$ in $G_{K}^{[2]}$, (c) 1-LD with density $\frac{4}{15}$ in $G_{K}^{[3]}$, (d) 1-Id with density $\frac{2}{9}$ in $G_{K}$.

For $r=1$, the strips have been investigated. Note that $G_{K}^{[2]}$ is not 1-twin-free.

Theorem $39([67])$ (a) We have $\partial_{1}^{I d}\left(G_{K}^{[3]}\right)=\frac{1}{3} ; \partial_{1}^{I d}\left(G_{K}^{[4]}\right)=\frac{5}{16}=0.3125 ; \partial_{1}^{I d}\left(G_{K}^{[5]}\right)=\frac{4}{15} \approx$ $0.26667 ; \partial_{1}^{I d}\left(G_{K}^{[6]}\right)=\frac{5}{18} \approx 0.27778$.

(b) For $k \geqslant 7$, we have $\frac{2}{9}+\frac{8}{81 k} \leqslant \partial_{1}^{I d}\left(G_{K}^{[k]}\right) \leqslant\left\{\begin{array}{l}\frac{2}{9}+\frac{6}{18 k}: k=0 \bmod 3 \\ \frac{2}{9}+\frac{8}{18 k}: k=1 \bmod 3 \\ \frac{2}{9}+\frac{7}{18 k}: k=2 \bmod 3\end{array}\right.$ 


\subsubsection{The Hexagonal Grid}

The hexagonal grid, $G_{H}$, has vertex set $V_{H}=\mathbb{Z} \times \mathbb{Z}$ and edge set $E_{H}=\{u v: u=(i, j)$ and $\left.u-v \in\left\{\left(0,(-1)^{i+j+1}\right),(1,0)\right\}\right\}$. It is the grid for which one has the sparsest and weakest results. Figure 6.10 will give constructions proving the upper bounds for Ths. 40 and 41.

- Locating-dominating codes

Theorem 40 ([113]) We have $\partial_{1}^{L D}\left(G_{H}\right)=\frac{1}{3}$.

- Identifying codes

The following upper bound is from [56], the lower bound from [61].

Theorem 41 We have $\frac{5}{12} \approx 0.41667 \leqslant \partial_{1}^{I d}\left(G_{H}\right) \leqslant \frac{3}{7} \approx 0.42857$.

(a)

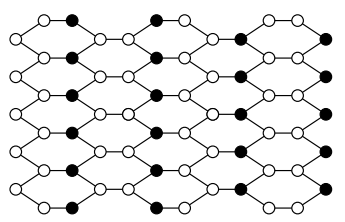

(b)

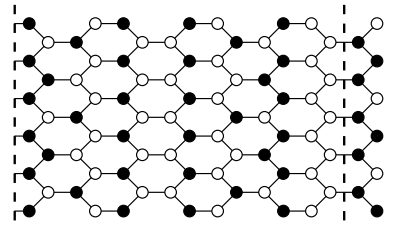

Fig. 6.10 Black vertices are the elements of a periodic code in $G_{H}$, which is (a) 1-LD with density $\frac{1}{3}$, (b) 1 -Id with density $\frac{3}{7}$.

In the following theorem, the lower bounds in (a) and (b) come from [131] and [144], respectively, and both upper bounds from [38]; the general lower bounds in (c) come from [33], and the upper bounds from [174].

Theorem 42 (a) We have $\partial_{2}^{I d}\left(G_{H}\right)=\frac{4}{19} \approx 0.21053$.

(b) We have $\frac{3}{25}=0.12 \leqslant \partial_{3}^{I d}\left(G_{H}\right) \leqslant \frac{1}{6} \approx 0.16667$.

(c) For $r \geqslant 8$, we have $\left.\left.\frac{\frac{2}{5 r+3}: r \text { even }}{\frac{2}{5 r+2}: r \text { odd }}\right\}\right\} \leqslant \partial_{r}^{I d}\left(G_{H}\right) \leqslant\left\{\begin{array}{ll}\frac{5 r+3}{6 r(r+1)}: r \text { even } \\ \frac{5 r^{2}+10 r-3}{(6 r-2)(r+1)^{2}}: r \text { odd }\end{array}\right.$. When $r$ increases, these bounds are close to $\frac{2}{5 r}$ and $\frac{5}{6 r} \approx \frac{0.83333}{r}$.

There are many better upper bounds, obtained by the use of heuristics, in [38] for $r \leqslant 30$.

\subsubsection{Paths and Cycles}

For $r=1$, the values of $L D\left(P_{n}\right), L D\left(C_{n}\right), \operatorname{Id}\left(P_{n}\right)$ and $\operatorname{Id}\left(C_{n}\right)$ have been completely determined.

Theorem 43 ([169]), ([170]) For every path or cycle $G_{n}$ of order $n \geqslant 1$, we have $L D\left(G_{n}\right)=\left\lceil\frac{2 n}{5}\right\rceil$.

Proof. For the lower bound, we give an alternative by Bertrand et al. [16] in 2004 to the 1988 proof by Slater. This proof uses the following counting argument, which can be applied to graphs other than paths or cycles, and can be adapted to Id codes, cf. proof of Th. 32. Let $C$ be a 1-LD code 
in $G_{n}$. Now $n-|C|$ non-codewords must be dominated by $C$, at most $|C|$ of these are dominated by one codeword, and the remaining are dominated by at least two codewords. Therefore,

$$
1 \times|C|+2 \times(n-2|C|) \leqslant \sum_{c \in C}|N(c)| .
$$

For paths and cycles, $|N(c)| \leqslant 2$, which leads to $|C| \geqslant\lceil 2 n / 5\rceil$. This lower bound is met with equality, see Fig. 6.11(a) for paths; for cycles, simply link the leftmost and rightmost vertices in the same figure.

(a)

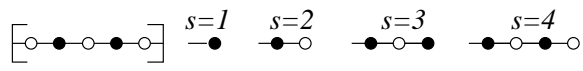

(b)

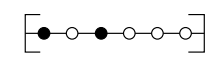

Fig. 6.11 Black vertices in the path with $n$ vertices are codewords. (a) $n=5 k+s, 0 \leqslant s<5$ : repeat the pattern between brackets $k-1$ times to the left and paste the appropriate tail to the right, to obtain a 1-LD code. (b) $n=6 k+s, 0<s \leqslant 6$ : repeat the pattern $k-1$ times to the left, then paste appropriate tails, not given here, to the left and to the right, to obtain a $2-\mathrm{LD}$ code.

Theorem $44([94])$ For every path $P_{n}$ of order $n \geqslant 3$, we have $\operatorname{Id}\left(P_{n}\right)=\left\lceil\frac{n+1}{2}\right\rceil$. For every cycle $C_{n}$ of length $n \geqslant 6$, we have $\operatorname{Id}\left(C_{n}\right)=3\left\lceil\frac{n}{2}\right\rceil-n$, and $\operatorname{Id}\left(C_{4}\right)=\operatorname{Id}\left(C_{5}\right)=3$.

It is then immediate that the best density in an infinite path is $2 / 5$ for 1 -LD codes and $1 / 2$ for 1 -Id codes (cf. Ths. 27(b) and 30(a), respectively). For $r>1$, it is not difficult to establish that these densities do not depend on $r$ and are $1 / 3$ and $1 / 2$ respectively [16]. But the general case $r>1$ for finite paths and cycles is surprisingly difficult and the problem is not settled yet for LD codes.

\subsubsection{Paths with $r>1$}

\section{- Locating-dominating codes}

Theorem 45 ([16]) Let $r \geqslant 2$. For all $n \geqslant 1$, we have $\frac{n+1}{3} \leqslant L D_{r}\left(P_{n}\right)$; for all $n \geqslant 2 r+1$, we have $L D_{r}\left(P_{n}\right) \leqslant\left\lceil\frac{n+7 r+6}{3}\right\rceil$.

For infinitely many values of $n$, the above upper bound can be improved to $\frac{n+r+2}{3}$, see [16] and [108]; the latter also completely solves the case $r=2$. Figure 6.11(b) gives the pattern for the upper bound in the following result.

Theorem 46 ([108]) For all $n \geqslant 1$, we have $L D_{2}\left(P_{n}\right)=\left\lceil\frac{n+1}{3}\right\rceil$.

In [71], the exact values of $L D_{3}\left(P_{n}\right)$ and $L D_{4}\left(P_{n}\right)$ are given for $n \geqslant 1$, the exact value of $L D_{r}\left(P_{n}\right)$ is given for $r \geqslant 5,1 \leqslant n \leqslant 7 r+3$, and the following is proved.

Theorem 47 ([71]) For $r \geqslant 5$ and $n \geqslant 3 r+2+6 r((r-3)(2 r+1)+r)$, we have $L D_{r}\left(P_{n}\right)=\left\lceil\frac{n+1}{3}\right\rceil$.

\section{- Identifying codes}

Theorem 48 ([16]) Let $r \geqslant 2$. For all $n \geqslant 2 r+1$, we have $\frac{n+1}{2} \leqslant I d_{r}\left(P_{n}\right)$, and this bound can be achieved for infinitely many values of $n$. 
The case $r=2$ is completely settled in [160]. And in 2011-12, the complete results were given independently by Chen, Lu \& Miao [52] and by Junnila \& Laihonen [130].

Theorem 49 ([52]), ([130]) Let $r \geqslant 2$ and $n=(2 r+1) p+q, p \geqslant 1,0 \leqslant q<2 r+1$.

(a) If $q=0$, then $I_{r}\left(P_{n}\right)=\frac{(2 r+1) p}{2}+1$ if $p$ is even; $I_{r}\left(P_{n}\right)=\frac{(2 r+1)(p-1)}{2}+2 r$ if $p$ is odd.

(b) If $1 \leqslant q \leqslant r+1$, then $I d_{r}\left(P_{n}\right)=\frac{(2 r+1) p}{2}+q$ if $p$ is even; $I d_{r}\left(P_{n}\right)=\frac{(2 r+1)(p-1)}{2}+2 r+1$ if $p$ is odd.

(c) If $r+2 \leqslant q \leqslant 2 r$, then $I d_{r}\left(P_{n}\right)=\frac{(2 r+1) p}{2}+q-1$ if $p$ is even; $I d_{r}\left(P_{n}\right)=\frac{(2 r+1)(p-1)}{2}+2 r+1$ if $p$ is odd.

This means that $I d_{r}\left(P_{n}\right)$ lies between $\frac{n+1}{2}$ and $\frac{n}{2}+r$, according to the values of $p$ and $q$.

\subsubsection{Cycles with $r>1$}

\section{- Locating-dominating codes}

Theorem 50 ([16]) Let $r \geqslant 2$. For all $n \geqslant 1$, we have $\frac{n}{3} \leqslant L D_{r}\left(C_{n}\right)$, and this bound can be achieved for infinitely many values of $n$.

In [52], the exact value of $L D_{2}\left(C_{n}\right)$ is given for $n \geqslant 1$, and in [72], the same is done for $L D_{3}\left(C_{n}\right)$ and $L D_{4}\left(C_{n}\right)$; furthermore, the exact values of $L D_{r}\left(C_{n}\right)$ are given for $r \geqslant 5, n \in[3,2 r+3] \cup\{3 r, 3 r+3\}$, and the following is proved.

Theorem 51 ([72]) Let $r \geqslant 5$ and $n \geqslant 12 r+5+6 r((r-3)(2 r+1)+r-1)$. Then we have $L D_{r}\left(C_{n}\right)=\left\lceil\frac{n}{3}\right\rceil$ if $n \neq 3 \bmod 6$, and $\frac{n}{3} \leqslant L D_{r}\left(C_{n}\right) \leqslant \frac{n}{3}+1$ otherwise.

The previous result is improved in [70]: the bound on $n$ becomes a polynomial in $r^{2}$ instead of $r^{3}$.

\section{- Identifying codes}

The crucial following lower bound is from [64] and solves the case $n$ even.

Theorem 52 ([64]), ([65]) For all $r \geqslant 2$ and $n \geqslant 2 r+2$, we have $\operatorname{Id}_{r}\left(C_{n}\right) \geqslant \operatorname{gcd}(2 r+1, n) \times$ $\left\lceil\frac{n}{2 \operatorname{gcd}(2 r+1, n)}\right\rceil$. If $n \geqslant 2 r+4$ is even, then $I d_{r}\left(C_{n}\right)=\frac{n}{2}$, and $I d_{r}\left(C_{2 r+2}\right)=2 r+1$.

Then [99] provides cases when the exact value of $I d_{r}\left(C_{n}\right)$ is known. The case $r=2$ is completely settled in [160]. More cases giving the exact value for $I d_{r}\left(C_{n}\right)$ are given in [178], further results of this type are given in [52], and finally in 2012, Junnila \& Laihonen [130] closed the case: for all $r \geqslant 2$ and $n \geqslant 2 r+2, I d_{r}\left(C_{n}\right)$ is known; but the many cases do not allow to give the results in a compact way. Let us simply mention here that $I d_{r}\left(C_{n}\right)$ lies between $\frac{n+1}{2}$ and $\frac{n}{2}+r$.

\subsubsection{Trees}

The following upper bound comes from [20], the lower bound from [166].

Theorem 53 Let $T$ be a tree of order $n$ with $\ell$ leaves and $s$ support vertices.

(a) We have $\frac{n+2(\ell-s)+1}{3} \leqslant L D_{1}(T) \leqslant \frac{n+\ell-s}{2}$.

(b) $([153])$ We have $L D_{1}(T) \leqslant \frac{2 n+3 \ell-2}{5}$. 
Moreover, the trees achieving the above bounds can be characterized. When $\ell=s$, the lower bound reads $L D_{1}(T)>n / 3$. The star $K_{1, n-1}$ has $s=1, \ell=n-1$ and $L D_{1}\left(K_{1, n-1}\right)=n-1$. All the intermediate values between $\left\lceil\frac{n+1}{3}\right\rceil$ and $n-1$ can be reached [166].

Theorem 54 ([26]) For any tree $T$ of order $n$, we have $L D_{2}(T)>\frac{n}{4}$, and $L D_{3}(T)>\frac{n}{5}$.

From this it is conjectured that for any tree $T$ of order $n, L D_{r}(T)>\frac{n}{r+2}$; if true, the conjecture is sharp [26]. The following upper bound comes from [94], the lower bound from [20].

Theorem 55 For any tree $T$ of order $n$ with $\ell$ leaves and s support vertices, we have

(a) $\frac{n+2 \ell-2}{2} \geqslant I d_{1}(T) \geqslant \frac{3(n+\ell-s+1)}{7}$. Both bounds are sharp.

(b) $([154]) \frac{3 n+2 \ell-1}{5} \geqslant I d_{1}(T) \geqslant \frac{2 n-s+3}{4}$.

The trees achieving the above bounds in (b) can be characterized. All the intermediate values between $\left\lceil\frac{3(n+1)}{7}\right\rceil$ and $n-1$ can be reached [41].

The following result corrects and completes the study of complete (or balanced) $q$-ary trees for 1-Id codes in [134], and also settles the case of 1-LD codes. It is illustrated by Fig. 6.12.

Theorem 56 ([17]) Let $h \geqslant 1, q \geqslant 2, C T_{h}^{q}$ be the complete $q$-ary tree of depth $h$, and $\xi_{h}^{q}=\frac{q^{h}-1}{q-1}$ be its order.

(a) We have $\operatorname{LD}\left(C T_{h}^{q}\right)=\left\lceil\frac{q^{2} \xi_{h}^{q}}{q^{2}+q+1}\right\rceil$.
(b) We have $\operatorname{Id}\left(C T_{h}^{2}\right)=\left\lceil\frac{20 \xi_{h}^{q}}{31}\right\rceil$; if $q>2$, then $\operatorname{Id}\left(C T_{h}^{q}\right)=\left\lceil\frac{q^{2} \xi_{h}^{q}}{q^{2}+q+1}\right\rceil$.

(a)

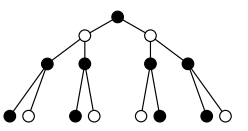

(b)

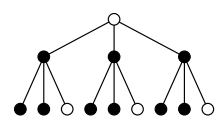

Fig. 6.12 Codewords are in black. (a) The complete binary tree of depth 4, with an optimal 1-LD code (of size 9) which is not 1-Id. (b) The complete ternary tree of depth 3, with an optimal 1-Id code (of size 9). This code is also an optimal 1-LD code.

Comparing (a) and (b) above, one can see that the complete nonbinary trees give yet another example of graphs in which 1-identification costs the same as 1-locating-domination - see Th. 21(b).

Although the computing problems of finding optimal $r$-LD and $r$-Id codes are generally NP-hard, even for some restricted classes of graphs (see Sect. 6.8 below), trees (and forests) form a class for which polynomial and even linear algorithms exist that output an optimal 1-LD or 1-Id code: see [169] for LD codes, [4] for Id codes, and [32] for Id codes in oriented trees.

\subsubsection{The $q$-ary $n$-Cube}

Here, the standard notation $n$ is not for the order of the cube, but for its dimension: the $q$-ary $n$-cube is denoted $\mathbb{F}_{q}^{n}$, where $\mathbb{F}_{q}=\{0,1, \ldots, q-1\}$; it has $q^{n} q$-ary vectors $v_{1} v_{2} \ldots v_{n}$, and the distance is usually the Hamming distance $d_{H}$ defined by $d_{H}(u, v)=\left|\left\{i: u_{i} \neq v_{i}, 1 \leqslant i \leqslant n\right\}\right|$. In this setting, $r$-dominating codes are rather called $r$-covering codes, and constitute an important topic 
inside coding theory. Their strong algebraic structure makes them very particular: for instance, a code may be a vector subspace of dimension $k$ in $\mathbb{F}_{q}^{n}$, in which case it has size $q^{k}$ and is said to be linear. Since a generator matrix with $k$ rows suffices to describe the code, this affects the size of an instance when considering complexity issues, see, e.g., [143], [91], [118], [119]. See also [55] for an overview of covering codes, with tables giving bounds on the sizes of optimal codes, linear or not, for the first values of $r$ and $n$. We shall restrict ourselves to the case $q=2$, although there are some works on the nonbinary cube, and denote $\mathbb{F}_{2}^{n}$ simply $\mathbb{F}^{n}$. Almost all the results given below concern identification. There are strong links between Id codes and $\mu$-fold coverings (where each vertex is dominated by at least $\mu$ codewords), but we do not have enough space to discuss them here; see, e.g., [76]. The following theorem connects the 1-LD number in $\mathbb{F}^{n}$ and the 2-domination number in $\mathbb{F}^{n-1}$.

Theorem 57 ([116]) For all $n \geqslant 5$, we have $\frac{n^{2} 2^{n+1}}{n^{3}+2 n^{2}+3 n-2} \leqslant L D_{1}\left(\mathbb{F}^{n}\right) \leqslant(2 n-3) \gamma_{2}\left(\mathbb{F}^{n-1}\right)$.

The exact values of $L D_{1}\left(\mathbb{F}^{n}\right)$ are known for $1 \leqslant n \leqslant 6$; for instance, $\left\{u \in \mathbb{F}^{5}: d_{H}(u, 00000)=1\right.$ or 4$\}$ is an optimal 1-LD code (of size 10) [116]. See also [73], [133]. The following result gives the complete answer for linear codes.

Theorem 58 ([116]) Let $n=3 \times 2^{k}-5+s$, for $k \geqslant 1$ and $0 \leqslant s<3 \times 2^{k}$. Then the size of an optimal 1-LD linear code in $\mathbb{F}^{n}$ is $2^{n-k}$.

We now turn to Id codes. The following theorem links the $r$-Id and $(2 r)$-domination numbers.

Theorem 59 ([134]) For $n \geqslant 3$, we have $\frac{n 2^{n+1}}{n(n+1)+2} \leqslant I d\left(\mathbb{F}^{n}\right)$ and $2^{n+1} /\left(1+\sum_{j=0}^{r}\left(\begin{array}{c}n \\ j\end{array}\right)\right) \leqslant I d_{r}\left(\mathbb{F}^{n}\right)$. If $r<n / 2$, then $\operatorname{Id}_{r}\left(\mathbb{F}^{n}\right) \leqslant\left(\begin{array}{l}n \\ r\end{array}\right) \gamma_{2 r}\left(\mathbb{F}^{n}\right)$.

The above lower bound on $I d_{r}\left(\mathbb{F}^{n}\right)$ is improved in [77] (but both bounds coincide for $r$ fixed and $n$ large enough), then in [75]. The next result uses the direct sum construction (DSC) $C \oplus \mathbb{F}^{s}=\{c \mid u$ : $\left.c \in C \subseteq \mathbb{F}^{n}, u \in \mathbb{F}^{s}\right\}$, where $\mid$ stands for concatenation. This is a classical tool, often used in coding theory, allowing to go from $\mathbb{F}^{n}$ to $\mathbb{F}^{n+s}, s \geqslant 1$.

Theorem 60 ([18]) For $n \geqslant 2$, we have $\operatorname{Id}\left(\mathbb{F}^{n+2}\right) \leqslant 4 I d\left(\mathbb{F}^{n}\right)$. If $C$ is an optimal 1-Id code in $\mathbb{F}^{n}$ such that $d_{H}(c, C \backslash\{c\})=1$ for every $c \in C$, then $\operatorname{Id}\left(\mathbb{F}^{n+1}\right) \leqslant 2 \operatorname{Id}\left(\mathbb{F}^{n}\right)$.

Since $\mathbb{F}^{s}$ is a 0 -Id code of size $2^{s}$, a generalization of the previous result would be the following conjecture: $I d_{r_{1}+r_{2}}\left(\mathbb{F}^{n_{1}+n_{2}}\right) \leqslant I d_{r_{1}}\left(\mathbb{F}^{n_{1}}\right) \times I d_{r_{2}}\left(\mathbb{F}^{n_{2}}\right)$. The case $r_{1}=r_{2}=1$ is proved in [76], which by iteration leads to

$$
I d_{r}\left(\mathbb{F}^{\sum_{i=1}^{r} n_{i}}\right) \leqslant \prod_{i=1}^{r} I d_{1}\left(\mathbb{F}^{n_{i}}\right) .
$$

Also, by refining the condition on $C$ in Th. 60 , one can widen the possibilities of the DSC, see, e.g., [76], [74], [31]. But $\operatorname{Id}\left(\mathbb{F}^{n+1}\right) \leqslant 2 \operatorname{Id}\left(\mathbb{F}^{n}\right)$ remains a conjecture; to our knowledge, the closest result obtained so far is the following.

Theorem 61 ([74]) For $n \geqslant 2$, we have $\operatorname{Id}\left(\mathbb{F}^{n+1}\right) \leqslant\left(2+\frac{1}{n+1}\right) \operatorname{Id}\left(\mathbb{F}^{n}\right)$.

There are also asymptotic results (with $n$ going to infinity) in [134], [118], [74], or [125].

In 2010, Charon et al. [31] gave tables with bounds on $I d_{r}\left(\mathbb{F}^{n}\right), 1 \leqslant r \leqslant 5, n \leqslant 21$; the same was done for $r \in\{2,3\}$ and $n \leqslant 30$ by Exoo et al. [75] in the same year; all sources are given. The lower bounds stem from the bounds discussed above, and from more ad hoc methods using the topology 
of the cube and studying local situations, possibly with the help of a computer. The upper bounds use widely the DSC, often enhanced by computer, or heuristics such as simulated annealing or the noising method.

In the linear case, the complete answer is known for $r=1$ (see [157] for $r>1$ ), and is very similar to Th. 58 for 1-LD codes. In particular, it uses the second part of Th. 60, which always works with linear codes and allows to go from $\mathbb{F}^{n}$ to $\mathbb{F}^{n+1}$ by doubling the size of the code.

Theorem 62 ([156]) Let $n=3 \times 2^{k}-3+s$, for $k \geqslant 1$ and $0 \leqslant s<3 \times 2^{k}$. Then the size of an optimal 1-Id linear code in $\mathbb{F}^{n}$ is $2^{n-k}$.

In conclusion, we offer an open problem about the monotonicity of $\operatorname{Id}_{r}\left(\mathbb{F}^{n}\right)$. It is true that for all $n \geqslant 2$, we have $I d_{1}\left(\mathbb{F}^{n}\right) \leqslant I d_{1}\left(\mathbb{F}^{n+1}\right.$ ) (Moncel [145] in 2006), but this is not the case for all $r$. For instance, $I d_{5}\left(\mathbb{F}^{6}\right)=63, I d_{5}\left(\mathbb{F}^{7}\right) \in\{31,32\}, I d_{5}\left(\mathbb{F}^{8}\right) \in[19,21], I d_{5}\left(\mathbb{F}^{9}\right) \in[12,17]$. We conjecture however that for a fixed $r>1$, there exists $n(r)$ such that for all $n \geqslant n(r)$, we have $I d_{r}\left(\mathbb{F}^{n}\right) \leqslant I d_{r}\left(\mathbb{F}^{n+1}\right)$.

\subsubsection{Bipartite Graphs and Discriminating Codes}

There are scattered results regarding bipartite graphs (see Ths. 15 and 100(c), Sect. 6.6.2.3, and the second sentence after Th. 107), but these graphs are also of interest to us because they lend themselves to the natural definition of discriminating codes: consider a bipartite graph $G=(I \cup$ $A, E)$ where $I$ represents individuals and $A$ their attributes (hair color, age, glasses, ...). A code $C \subseteq A$ is discriminating if every individual has at least one attribute in $C$, and no two individuals have the same set of attributes in $C[30]$.

Theorem 63 ([28]) If $C$ is an optimal discriminating code in $G$, then $\left\lceil\log _{2}(|I|+1)\right\rceil \leqslant|C| \leqslant|I|$. Both bounds are sharp.

Discriminating codes may be seen as a generalization of 1-Id codes, since a 1-Id code in a graph $G=(V, E)$ is clearly a discriminating code in the bipartite graph consisiting of $V$ on the one hand, and vertices representing the closed neighborhoods in $G$ on the other hand, with edges linking each closed neighborhood to its members.

The definition can be extended to any odd $r \geqslant 1$, by asking that for $i \in I, i_{1} \in I, i_{2} \in I, i_{1} \neq i_{2}$, we have $N_{r}(i) \cap C \neq \emptyset$ and $N_{r}\left(i_{1}\right) \cap C \neq N_{r}\left(i_{2}\right) \cap C$. The associated decision problem is NP-complete for any fixed $r \geqslant 1$ [28], but for $r=1$ there is a linear algorithm in the case of trees [30].

Special cases of bipartite graphs have been investigated: the infinite square and hexagonal grids [28], bipartite planar graphs [30], and the binary $n$-cube, for which it can be proved that for any odd $r$, there is a bijection between the set of $r$-Id codes in $\mathbb{F}^{n}$ and the set of $r$-discriminating codes in $\mathbb{F}^{n+1}[29]$.

\subsubsection{Line Graphs and Edge Identification}

We can identify an edge with edges in a graph $G=(V, E)$ : for $e \in E$, we denote $N(e)$ the set of edges which are adjacent to $e$, and $N[e]=N(e) \cup\{e\}$. A code $C_{E} \subseteq E$ is edge-identifying 
if for every $e \in E$, we have $N[e] \cap C_{E} \neq \emptyset$, and for every $e_{1} \in E, e_{2} \in E, e_{1} \neq e_{2}$, we have $N\left[e_{1}\right] \cap C_{E} \neq N\left[e_{2}\right] \cap C_{E}$. Clearly, $C_{E}$ is edge-identifying in $G$ if and only if, in the line graph $L(G)$, the vertices corresponding to the edges in $C_{E}$ form a 1-Id code. This motivates the study of Id codes in the class of line graphs. For instance, one has the following results.

Theorem 64 ([81]) (a) If $G$ is a 1-twin-free line graph of order $n$, then $\operatorname{Id}(G)>\frac{3 \sqrt{2}}{4} \sqrt{n} \approx$ $1.06066 \sqrt{n}$.

(b) For $n \geqslant 4$, the minimum size of an edge-identifying code in the binary cube $\mathbb{F}^{n}$ is $2^{n-1}$.

The decision problem associated to edge identification is NP-complete [148].

\subsubsection{Miscellaneous}

\section{- Planar and outerplanar graphs}

Theorem 65 ([155]) For any planar graph $G$ of order $n \geqslant 18$, we have $L D(G) \geqslant \frac{n+10}{7}$; for any outerplanar graph $G$ of order $n$, we have $L D(G) \geqslant \frac{2 n+3}{7}$. Both bounds are sharp.

\section{- Split graphs}

Split graphs have been studied in several works; in particular, complete suns have been intensively investigated, both for 1-LD and 1-Id codes: see, e.g., [2], [122].

- In the context of LD and Id codes, attention has been given in the existing literature to the following families: graph products (mostly Cartesian, but also corona, lexicographic, Kronecker or direct), cubic graphs, rotagraphs and fasciagraphs, finite rectangles extracted from the infinite grids ( $k_{1}$ rows, $k_{2}$ columns), $n$-dimensional infinite grids, Kneser graphs, circulant networks, complementary prisms, the $n$-cube endowed with the Lee metric, triangle-free graphs, cographs, Sierpiński graphs, interval graphs, permutation graphs, triangular graphs, vertex-transitive graphs, fractal graphs, block graphs, chordal graphs, series parallel graphs, and others. Random graphs have also been studied.

See also Tables 1.3-1.5 in [79] for a survey of the different lower and upper bounds, and their tightness, for different classes of graphs.

\subsection{Relationships with Other Parameters}

We study the different relationships linking the LD and Id numbers of a graph to different parameters such as maximum and minimum degree, girth, domination number, independent domination numbers, or diameter, mostly for $r=1$.

\subsubsection{Locating-Dominating Codes}

Theorem 66 ([170]) For any graph $G$ of order $n \geqslant 2$ and maximum degree $\Delta$, we have $n \leqslant$ $L D_{1}(G)+\sum_{i=1}^{\Delta}\left(\begin{array}{c}L D_{i}(G) \\ i\end{array}\right)$ 
For $\Delta=2$ (paths and cycles), this can be improved to $n \leqslant \frac{5}{2} L D_{1}(G)$, see Th. 43 .

Theorem 67 below gives upper bounds on $L D_{1}(G)$, with conditions on minimum degree and girth, then the best known constructions, for $n$ small or arbitrarily large.

Theorem 67 Let $G$ be a graph of order $n$, minimum degree $\delta$, and girth $g \geqslant 5$.

$\left(a_{1}\right)([50])$ If $\delta \geqslant 2$, then $L D_{1}(G) \leqslant \frac{n}{2}$.

(a $\left.a_{2}\right)([10])$ If $\delta=3$, then $L D_{1}(G) \leqslant \frac{22 n}{45} \approx 0.4889 n$.

(b) ([10]) The cycle $C_{6}$ has $n=6, \delta=2, g=6$, and $L D_{1}\left(C_{6}\right)=3=\frac{n}{2}$; there are infinitely many connected graphs $G$ of order $n$ with $\delta=2, g=5$, and $L D_{1}(G)=\frac{n-1}{2}$.

The Heawood graph $H_{14}$ (see Fig. 6.13(a)) has $n=14, \delta=3, g=6$, and $L D_{1}\left(H_{14}\right)=6=$ $\frac{3 n}{7} \approx 0.4286 n$; there are infinitely many connected graphs $G$ of order $n$ with $\delta=3, g=5$, and $L D_{1}(G)=\frac{4(n-1)}{11} \approx 0.3636 n$.

(a)

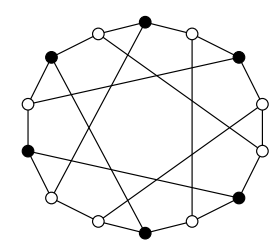

(b)

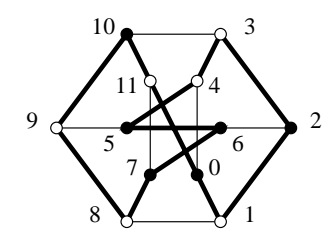

Fig. 6.13 Black vertices form optimal codes. (a) The Heawood graph $H_{14}$; (b) The graph $G_{12}$.

We recall that $i(G)$ (respectively, $\beta(G)$ ) denotes the minimum (respectively, maximum) cardinality of an independent dominating set in a graph $G$.

Theorem 68 ([50]) (a) If $T$ is a nontrivial tree, then $i(T) \leqslant L D_{1}(T) \leqslant \beta(T)$.

(b) For every nontrivial tree $T$, we have $L D_{1}(T)+\gamma_{1}(T) \leqslant n$, with equality if and only if $T$ is a tree consisting only of leaves and support vertices.

Theorem 69 ([25]) Let $G$ be a graph of order $n$ and diameter $D \geqslant 3$. Then $L D_{1}(G) \leqslant n-\left\lceil\frac{3 D-1}{5}\right\rceil$, and the bound is tight. Consequently, if $L D_{1}(G) \in\{n-2, n-1\}$, then $G$ has diameter at most 3 .

\subsubsection{Identifying Codes}

We first give a lower bound involving the maximum degree, then discuss different upper bounds, first in terms of maximum degree, then of minimum degree and girth.

Theorem 70 ([134]) Let $r \geqslant 1$. If $G$ is an $r$-twin-free graph of order $n$ and maximum degree $\Delta$, then $I d_{r}(G) \geqslant \frac{2 n}{\Delta+2}$.

For $r=1$, [79] gives a full characterization of the graphs reaching the previous lower bound.

Theorem $71([83])$ Let $r \geqslant 1$ and $G$ be a connected $r$-twin-free graph of order $n$ and maximum degree $\Delta$. Then $I d_{r}(G) \leqslant n-\frac{n(\Delta-2)}{\Delta(\Delta-1)^{5 r}-2}=n-\frac{n}{\Theta\left(\Delta^{5 r}\right)}$. 
It is conjectured in [84] that there exists a constant $c$ such that, for every nontrivial connected 1-twin-free graph $G$, we have $I d_{1}(G) \leqslant n-\frac{n}{\Delta}+c$. Graphs such that $I d_{1}(G)=n-\frac{n}{\Delta}$ exist (e.g., $K_{\Delta, \Delta}$ with $\Delta \geqslant 3$ ). The complete $q$-ary trees $T$ have $\Delta=q+1$ and, by $T h$. 56(b), we have $I d_{1}(T)=\left\lceil n-\frac{n}{\Delta-1+1 / \Delta}\right\rceil$. The conjecture holds for graphs with very high $\Delta$, and for $\Delta=2$, and the following results are intended to further substantiate it.

Theorem 72 ([84]) Let $G$ be a connected, 1-twin-free, triangle-free graph with order $n$ and maximum degree $\Delta \geqslant 3$.

(a) We have $I d_{1}(G) \leqslant n-\frac{n}{\Delta+\frac{3 \Delta}{\ln \Delta-1}}=n-\frac{n}{\Delta+o(\Delta)}$.

(b) If moreover $G$ is a nontrivial planar or bipartite graph, then $I d_{1}(G) \leqslant n-\frac{n}{\Delta+9}$.

Other graphs can be exhibited, with $I d_{1}(G) \leqslant n-\frac{n}{o(\Delta)}$. In the following theorem, a vertex $z$ is said to be forced if there are two vertices $u$ and $v$ with symmetric difference $N[u] \Delta N[v]$ equal to $\{z\}$, which implies that $z$ belongs to any 1-Id code in $G$.

Theorem 73 ([89]) (a) Let $G$ be a connected, 1-twin-free graph, with order $n$ and maximum degree $\Delta \geqslant 3$. Then $I d_{1}(G) \leqslant n-\frac{n f^{2}}{103 \Delta}$, where $f$ is the ratio over $n$ of the number of non-forced vertices. In particular, if $G$ is $\Delta$-regular, then $\operatorname{Id}_{1}(G) \leqslant n-\frac{n}{103 \Delta}=n-\frac{n}{\Theta(\Delta)}$.

(b) There exists an integer $\Delta_{0}$ such that for every connected, 1-twin-free graph with order $n$ and maximum degree $\Delta \geqslant \Delta_{0}$, we have $I d_{1}(G) \leqslant n-\frac{n}{103 \Delta(\Delta+1)^{2}}=n-\frac{n}{\Theta\left(\Delta^{3}\right)}$.

The following result can be compared to Th. 67 .

Theorem 74 ([10]) Let $G$ be a twin-free graph of order $n$, minimum degree $\delta$, and girth $g \geqslant 5$.

$\left(a_{1}\right)$ If $\delta \geqslant 2$, then $I d_{1}(G) \leqslant \frac{5 n}{7} \approx 0.7143 n$.

$\left(a_{2}\right)$ If $\delta=3$, then $I_{1}(G) \leqslant \frac{31 n}{45} \approx 0.6889 n$.

(b) The cycle $C_{7}$ has $n=7, \delta=2, g=7$, and $I d_{1}\left(C_{7}\right)=5=\frac{5 n}{7}$; there are infinitely many connected graphs $G$ of order $n$ with $\delta=2, g=5$, and $I d_{1}(G)=\frac{3(n-1)}{5}$.

The graph $G_{12}$ with vertex set $\left\{x_{i}: 0 \leqslant i \leqslant 11\right\}$ and the edges of the Hamiltonian cycle $x_{0} x_{1} \ldots x_{11} x_{0}$ plus the six edges $x_{0} x_{4}, x_{1} x_{8}, x_{2} x_{6}, x_{3} x_{10}, x_{5} x_{9}$ and $x_{7} x_{11}$ (see Fig. 6.13(b)) has $n=12, \delta=3, g=5$, and $I d_{1}\left(G_{12}\right)=6=\frac{n}{2}$; there are infinitely many connected graphs $G$ of order $n$ with $\delta=3, g=5$, and $I d_{1}(G)=\frac{5(n-1)}{11} \approx 0.4545 n$.

\subsubsection{Twin-Free Graphs and Parameters}

From now on and until the end of this section, we investigate the extremal values that the following parameters: size, order, maximum and minimum degree, size of a maximum clique, radius, diameter, size of a maximum independent set, and identification number, can achieve in a connected $r$-twinfree graph. If $\pi$ stands for such a parameter, we fix $r$ and search for the smallest value $f_{r}(\pi)$ that $\pi$ can reach, or we fix $r$ and $n$ and search for the smallest and largest values $f_{r, n}(\pi)$ and $F_{r, n}(\pi)$. Therefore, $f_{r}(\pi)=\min \{\pi(G): G$ connected, $r$-twin-free with at least $2 r+1$ vertices $\}$, $f_{r, n}(\pi)=\min \{\pi(G): G$ connected, $r$-twin-free with $n \geqslant 2 r+1\}$, and $F_{r, n}(\pi)$ is defined similarly. The first case, when $\pi=\varepsilon$ is the size of the graph, will help understand easily these notions.

- Number of edges $\varepsilon$

For any connected graph of order $n$, the size is between $n-1$ (trees) and $\frac{n(n-1)}{2}$ (the complete 
graph). The size of a connected $r$-twin-free graph of order at least $2 r+1$ is at least $2 r$, and if the order is $n \geqslant 2 r+1$, then this number is at least $n-1$; moreover, the paths $P_{2 r+1}$ and $P_{n}$ meet these bounds. So $f_{r}(\varepsilon)=2 r$ and $f_{r, n}(\varepsilon)=n-1$. The maximum number of edges possible for $r$-twin-free graphs of order $n$ is known for $r=1$, and can be achieved only by the complete graphs minus a maximum matching [64]. For $r>1$, we are close to the exact value.

Theorem 75 ([64]) For all $n \geqslant 3$, we have $F_{1, n}(\varepsilon)=\frac{n(n-1)}{2}-\left\lfloor\frac{n}{2}\right\rfloor$.

Theorem 76 ([5]) (a) For $n$ large enough, we have $\frac{n^{2}}{2}-2 n \log _{2} n \lesssim F_{2, n}(\varepsilon) \lesssim \frac{n^{2}}{2}-\frac{1}{2} n \log _{2} n$.

(b) For $r>2$ and $n$ large enough with respect to $r$, we have $\frac{n^{2}}{2}-r n \log _{2} n \lesssim F_{r, n}(\varepsilon) \lesssim \frac{n^{2}}{2}-$ $0.63(r-0.915) n \log _{2} n$.

For $r>2$, the gap between the above lower and upper bounds is about $(0.37 r+0.58) n \log _{2} n$. For $r=10$ and $n$ around 60000 , it represents $0.2 \%$.

- Number of vertices $n$

Obviously, $f_{r, n}(n)=F_{r, n}(n)=n$ for all $r \geqslant 1, n \geqslant 2 r+1$, and the following comes from Th. 2 .

Theorem 77 For all $r \geqslant 1$, we have $f_{r}(n)=2 r+1$.

- Maximum degree $\Delta$

In any connected graph of order $n$, the maximum degree is between 1 (if $n=2$ ) or 2 (the path, the cycle, $n \geqslant 3$ ), and $n-1$ (the complete graph, the star, every graph $G$ with $\gamma_{1}(G)=1$ ).

Theorem 78 ([45]) (a) For all $r \geqslant 1$, we have $f_{r}(\Delta)=2$; for all $r \geqslant 1$ and $n \geqslant 2 r+1$, we have $f_{r, n}(\Delta)=2$.

(b) For all $n \geqslant 3$, we have $F_{1, n}(\Delta)=n-1$.

(c) For all $p \geqslant 2$ and $n \geqslant 5$, if $2^{p-1}+p-1<n \leqslant 2^{p}+p$, then $F_{2, n}(\Delta)=n-p-1$.

(d) For all $r \geqslant 3$ and $n \geqslant 2 r+1$, we have $F_{r, n}(\Delta) \leqslant k$, where $k$ is the largest integer such that $k+(r-2)\left\lceil\log _{3}(k+1)\right\rceil+\left\lceil\log _{2}(k+1)\right\rceil \leqslant n-1$.

When $r>2$, there are too many cases for the lower bounds on $F_{r, n}(\Delta)$ to be given here. Let us only mention that: (i) we have the exact value of $F_{r, n}(\Delta)$ for infinitely many values of $n$ and $r$ - for instance, if $r=100$ and $60050 \leqslant n \leqslant 60143$, then $F_{100, n}(\Delta)=59048$; (ii) if $n$ is large with respect to $r$, the lower bound on $F_{r, n}(\Delta)$ approximately behaves like $n-r \log _{3} n$, whereas the upper bound in Case (d) above behaves like $n-\left(r-2+\log _{2} 3\right) \log _{3} n$, which gives a gap between the bounds roughly equal to $0.415 \times \log _{3} n$ (independent of $r$ ).

- Minimum degree $\delta$

In any connected graph of order $n$, the minimum degree is between 1 and $n-1$ (the complete graph). The first part of Case (b) below relies on the fact that $K_{n}$ minus a maximum matching is 1-twin-free, cf. Th. 75 .

Theorem 79 ([5]) (a) For all $r \geqslant 1$, we have $f_{r}(\delta)=1$; for all $r \geqslant 1$ and $n \geqslant 2 r+1$, we have $f_{r, n}(\delta)=1$.

(b) For all $n \geqslant 3$, we have $F_{1, n}(\delta)=n-2$; for all $n \geqslant 5$, we have $F_{2, n}(\delta)=\left\lfloor\frac{n-2}{2}\right\rfloor$.

(c) For all $r \geqslant 3$, we have $F_{r, 2 r+1}(\delta)=1$.

(d) Let $r \geqslant 3, n \geqslant 2 r+2$ and $k=\left\lfloor\frac{n-2}{r}\right\rfloor$.

$\left(d_{1}\right)$ We have $k-1 \leqslant F_{r, n}(\delta)$ if $k$ is odd, and $k \leqslant F_{r, n}(\delta)$ if $k$ is even.

$\left(d_{2}\right)$ For $r \in\{3,4,5, \ldots, 24\}$, we have $F_{r, n}(\delta) \leqslant \frac{n}{\left\lfloor\frac{r}{2}\right\rfloor+1}-1$.

$\left(d_{3}\right)$ For $r \geqslant 25$, we have $F_{r, n}(\delta) \leqslant \min \left\{\frac{n}{\left\lfloor\frac{r}{2}\right\rfloor+1}-1, \frac{3 n-r+2}{2(r-5)}\right\}$. 
We conjecture that $F_{r, n}(\delta)$ is close, possibly equal, to $\left\lfloor\frac{n-2}{r}\right\rfloor$.

\section{- Size $\omega$ of a maximum clique}

In any connected graph of order $n$, a maximum clique has size between 2 and $n$. The clique $K_{n}$ is far from being $r$-twin-free; actually any graph whose $r$-th power is the clique has $r$-twins. However, a twin-free graph may contain quite a large clique, and it is remarkable that we can exactly determine its size. Case (b) below is connected to Th. 95. Case (c) depends on some conditions on $k$ and $n$ that we do not give here.

Theorem 80 (Charon, Hudry \& Lobstein 2011 [44]) (a) For all $r \geqslant 1$, we have $f_{r}(\omega)=2$; for all $r \geqslant 1$ and $n \geqslant 2 r+1$, we have $f_{r, n}(\omega)=2$.

(b) For all $n \geqslant 3$, we have $F_{1, n}(\omega)=k$, where $k$ is the largest integer such that $k+\left\lceil\log _{2} k\right\rceil \leqslant n$.

(c) For all $r \geqslant 2$ and $n \geqslant 2 r+1$, let $k$ be the largest integer such that $k+r\left\lceil\log _{2} k\right\rceil \leqslant n-1$. Then, according to conditions on $n$ and $k$, we have $F_{r, n}(\omega)=k$ or $k+1$.

If $n$ is large with respect to $r$, then $F_{r, n}(\omega)$ behaves like $n-r \log _{2} n$. If $r$ is a fraction of $n$, then $F_{r, n}(\omega)$ is bounded by above by a constant; the extremal case is when $r=\frac{n-1}{2}$ and $F_{r, n}(\omega)=2$ (path $P_{2 r+1}$ ).

- Radius $\rho$

In any connected graph of order $n$, the radius is between 1 (the complete graph, the star) and $\left\lfloor\frac{n}{2}\right\rfloor$ (the path, the cycle). The study of the radius in twin-free graphs is easy, the results are complete.

Theorem 81 ([5]) (a) For all $r \geqslant 1$, we have $f_{r}(\rho)=r$; for all $r \geqslant 1$ and $n \geqslant 2 r+1$, we have $f_{r, n}(\rho)=r$.

(b) For all $r \geqslant 1$ and $n \geqslant 2 r+1$, we have $F_{r, n}(\rho)=\left\lfloor\frac{n}{2}\right\rfloor$.

- Diameter $D$

In any connected graph of order $n$, the diameter is between 1 (the complete graph) and $n-1$ (the path). The results on the diameter in twin-free graphs are complete. Figure 6.14 illustrates Case (c).

Theorem 82 ([5]) (a) For all $r \geqslant 1$, we have $f_{r}(D)=r+1$.

(b) For all $r \geqslant 1$, we have $f_{r, 2 r+1}(D)=2 r$.

(c) For all $r \geqslant 1$ and $n \geqslant 2 r+2$, we have $f_{r, n}(D)=r+1$.

(d) For all $r \geqslant 1$ and $n \geqslant 2 r+1$, we have $F_{r, n}(D)=n-1$.

Fig. 6.14 The case $n=$

$15, r=3$ : the graph

is 3 -twin-free and has

diameter $r+1=4$.

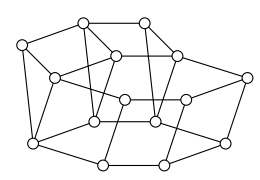

- Size $\alpha$ of a maximum independent set

In any connected graph of order $n, \alpha$ lies between 1 (the complete graph) and $n-1$ (the star). Theorem 89 contributes to Case (a) in the following theorem.

Theorem 83 ([5]) (a) For all $r \geqslant 1$, we have $f_{r}(\alpha)=r+1$.

(b) For all $n \geqslant 3$, we have $f_{1, n}(\alpha)=2$.

(c) For all $r \geqslant 2$, we have $f_{r, 2 r+1}(\alpha)=r+1$.

(d) Let $r \geqslant 2, n \geqslant 2 r+2$ and $k=\left\lfloor\frac{n-2}{r}\right\rfloor$. We have $r+1 \leqslant f_{r, n}(\alpha) \leqslant\left\{\begin{array}{l}\frac{2 n}{k+2}: k \text { even } \\ \frac{2 n}{k+1}: k \text { odd }\end{array}\right.$. 
The star gives Case (a) in the following theorem.

Theorem 84 ([5]) (a) For all $n \geqslant 3$, we have $F_{1, n}(\alpha)=n-1$.

(b) For all $r \geqslant 2$ and $n \geqslant 2 r+1$, we have $\max \left\{\left\lceil\frac{n}{2}\right\rceil, k+\left\lceil\log _{2} k\right\rceil\left\lfloor\frac{r}{2}\right\rfloor\right\} \leqslant F_{r, n}(\alpha) \leqslant n-r$, where $k$ is the largest integer such that $k+r\left\lceil\log _{2} k\right\rceil \leqslant n-1$.

If $n$ is large with respect to $r$, then $k+\left\lceil\log _{2} k\right\rceil\left\lfloor\frac{r}{2}\right\rfloor$ behaves approximately like $n-\frac{r}{2} \log _{2} n$.

- Identification number $I d_{r}$

We start with $F_{r, n}\left(I d_{r}\right)$ and $f_{r, n}\left(I d_{r}\right)$, and conclude with $f_{r}\left(I d_{r}\right)$, the most interesting of the three functions. From Sect. 6.2.1, in particular Ths. 8 and 13, we derive the following results.

Theorem 85 (a) For all $r \geqslant 1$, if $n=2 r+1$, or if $n \geqslant 3 r^{2}$ is even, or if $n \geqslant 3 r^{2}+1$ is odd, we have $F_{r, n}\left(I d_{r}\right)=n-1$. In particular, for all $n \geqslant 3$, we have $F_{1, n}\left(I d_{1}\right)=n-1$.

(b) For all $r \geqslant 1$ and $n \geqslant 2^{2 r}$, we have $f_{r, n}\left(\operatorname{Id}_{r}\right)=\left\lceil\log _{2}(n+1)\right\rceil$.

Theorem $86([44])$ (a) We have $f_{1}\left(I d_{1}\right) \geqslant 2$ and for all $r \geqslant 2, f_{r}\left(I d_{r}\right) \geqslant\left\lceil\log _{2}(2 r+4)\right\rceil$.

(b) For all $r \geqslant 1$, we have $f_{r}\left(I d_{r}\right) \leqslant r+1$.

The constructive upper bound is illustrated by Fig. 6.15 .
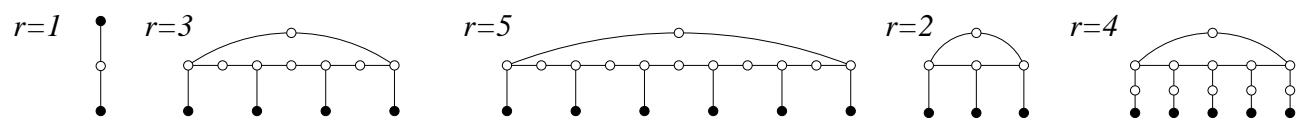

Fig. 6.15 Different graphs with optimal $r$-Id codes of size $r+1$. Codewords are in black. For $r \geqslant 3$ odd, there is a cycle $C_{2 r+2}$ and $n=(2 r+2)+r+1$. For $r$ even, there is a cycle $C_{r+2}$ and $n=(r+2)+\frac{r}{2}(r+1)$.

The lower and upper bounds in Th. 86 coincide for $r \in\{1,2,3\}$. For $r=4$, ad hoc arguments show that no graph admits a 4 -Id code of size 4 . Therefore,

Theorem 87 ([44]) For $r \in\{1,2,3,4\}$, we have $f_{r}\left(I d_{r}\right)=r+1$.

Open problem: for $r \geqslant 5$, find the exact value of $f_{r}\left(I d_{r}\right)$, knowing that it lies between $\left\lceil\log _{2}(2 r+4)\right\rceil$ and $r+1$.

\subsection{Structural Issues}

This section is mostly devoted to identifying codes, either because identifying codes lead to the problem of their very existence, i.e., to the important issue of when a graph is twin-free, or simply because in the literature, more attention has been devoted to these topics for identifying codes, compared to locating-dominating codes.

We shall study problems such as the existence of a chordless path in twin-free graphs, whether a twin-free graph deprived of a vertex remains twin-free, the consequence of adding or deleting an edge or a vertex on the identification number, or graphs that are critical, in some sense, with respect to locating-domination or identification.

Locating-dominating and identifying codes do not lend themselves well to Nordhaus-Gaddumtype bounds, and there are very few results. We give two examples. 
Theorem 88 ([106]) For any graph $G$ of order $n \geqslant 1$, we have $\left|L D_{1}(G)-L D_{1}(\bar{G})\right| \leqslant 1$.

Also, because $P_{4}=\bar{P}_{4}$ and $I d_{1}\left(P_{4}\right)=3$, the trivial bounds $2\left\lceil\log _{2}(n+1)\right\rceil \leqslant I d_{1}(G)+I d_{1}(\bar{G}) \leqslant$ $2(n-1)$ and $\left\lceil\log _{2}(n+1)\right\rceil^{2} \leqslant I d_{1}(G) \times I d_{1}(\bar{G}) \leqslant(n-1)^{2}$ are tight.

\subsubsection{Structural Properties of Twin-Free Graphs}

The first part of Th. 2 is an obvious consequence of the following result, due to Auger in 2008 [3], which is not obvious.

Theorem 89 Let $r \geqslant 1$ and $G$ be a connected $r$-twin-free graph. Then $G$ admits $P_{2 r+1}$, the path on $2 r+1$ vertices, as an induced subgraph, i.e., there is a chordless path of length $2 r$ in $G$.

\subsubsection{Deletion of a Vertex in a Twin-Free Graph}

Theorems 90-94, due to Charon et al. [35] in 2007, give results which vary widely with $r$. If $G=$ $(V, E)$ is $r$-twin-free, we say that $G$ is $r$-terminal if for all $v \in V, G-v$ is not $r$-twin-free (so $G$ is not $r$-terminal if there exists $v \in V$ such that $G-v$ is also $r$-twin-free). An alternative would be r-twin-free critical, see also the discussion before Th. 96.

If $n=2 r+1$, the only connected $r$-twin-free graph is the path $P_{2 r+1}$ for $r \geqslant 1$, and the only $r$-terminal graph is $P_{2 r+1}$ for $r>1$ (the case of $P_{3}$ is particular, because removing the middle vertex yields two isolated vertices, constituting a 1-twin-free graph). We address the following questions: (a) are there 1-terminal graphs? (b) For $r>1$, are there $r$-terminal graphs other than $P_{2 r+1}$ ?

The answer to (a) is negative, and the answer to (b) is multifold: it is negative if we restrict ourselves to trees; it is positive if $r \geqslant 3$. The case $r=2$ remains open.

- The case $r=1$

Theorem 90 ([35]) Let $n \geqslant 3$ and $G=(V, E)$ be any connected 1 -twin-free graph of order $n$. Then $G$ is not 1-terminal.

Proof. If $n=3$, then $G=P_{3}$ is not 1-terminal, as already seen; so we can assume that $n \geqslant 4$. By Th. 6 , there is a 1-Id code $C$ of size $n-1$ in $G$. Consider $G-v$, where $\{v\}=V \backslash C(G-v$ may be connected or not); then $C$ is still 1-identifying in $G-v$, because removing $v$ did not cut connexions of length $r(=1)$ between pairs of vertices not containing $v$ itself - this explains why the cases $r=1$ and $r>1$ are different. Therefore, $G-v$ is 1-twin-free.

The following theorem sharpens the previous one with respect to connectivity.

Theorem 91 ([35]) Let $n \geqslant 4$ and $G=(V, E)$ be any connected 1-twin-free graph of order $n$. Then there exists $v \in V$ such that $G-v$ is 1-twin-free and connected.

\section{- The case of trees}

Theorem 92 ([35]) Let $r \geqslant 1, n \geqslant 2 r+2$ and $T=(V, E)$ be any (connected) $r$-twin-free tree of order $n$. Then there exists a leaf $v \in V$ such that $T-v$ is $r$-twin-free (and connected). Consequently, the only $r$-terminal trees are the paths $P_{2 r+1}$, for $r>1$. 
- The case $r \geqslant 3$

We now consider general graphs, for $r \geqslant 3$.

Theorem 93 ([35]) For each integer $r \geqslant 3$, there is a graph $G, G \neq P_{2 r+1}$, which is $r$-terminal.

Sketch of proof. We search for a connected $r$-twin-free graph $G=(V, E)$, with $|V| \geqslant 2 r+2$, $r \geqslant 3$, such that for all $v \in V, G-v$ is not $r$-twin-free. One possible construction is the following: take a cycle of length $2 r$ with vertices $c_{i}(i \in\{0,1, \ldots, 2 r-1\})$, and add one vertex $s_{i}$ together with the edge $c_{i} s_{i}$ for every value of $i$ but one.

- Other values of $r$ and open problems

The construction of Th. 93 does not work for $r=2$. Other constructions have been tried and failed, and the problem remains open: Apart from $P_{5}$, do 2-terminal graphs exist?

Theorem 94 ([35]) For each integer $r \geqslant 6$, there are infinitely many $r$-terminal graphs.

Now, another open problem is the situation for $r \in\{3,4,5\}$ : there exist $r$-terminal graphs, but are they in finite or infinite number? Conversely, there is a bound on the number of vertices that need to be added in order to obtain a 1-twin-free graph.

Theorem 95 ([94]) If $G$ has order $n$, then $G$ embeds as an induced subgraph in a 1-twin-free graph with order at most $n+\left\lceil\log _{2} n\right\rceil$. The bound is tight.

\subsubsection{Deletion of an Edge in a Twin-Free Graph}

The graphs that we have called terminal in the previous section might as well have been called vertex-terminal, and the $r$-twin-free graphs $G=(V, E)$ such that for all $e \in E, G-e$ is not $r$-twinfree could then have been called $r$-edge-terminal. Back to 1974 however, the expression "line-critical point distinguishing graph" was coined in [68] (for $r=1$ only) and the following result was proved.

Theorem 96 ([68]) A nontrivial connected graph $G$ is line-critical point distinguishing [or 1-edgeterminal] if and only if $G=P_{3}$.

A nonempty graph is line-critical point distinguishing if and only if it is the disjoint union of paths of length two and isolated vertices.

To our knowledge, everything remains to be done for $r>1$.

\subsubsection{Adding or Deleting Edges or Vertices Can Save Codewords}

The question is to determine how the identification or locating-domination numbers change when we add or delete edges or vertices. We first deal with identifying codes.

\subsubsection{Identifying Codes: Adding or Deleting an Edge}

The problem is the following: given an $r$-twin-free graph $G=(V, E)$ and an edge $e \in E$, and assuming that the graph $G-e$ is $r$-twin-free, what can be said about the relationship between 
$I d_{r}(G)$ and $I d_{r}(G-e)$ ? The answer widely depends on $r$, as show the following two theorems by Charon et al. [37] in 2014.

- The case $r=1$

Theorem 97 If $G$ and $G-e$ are 1-twin-free, then $\operatorname{Id}(G)-I d(G-e) \in\{-2,-1,0,1,2\}$. Moreover, pairs of connected graphs $G$ and $G-e$ such that $\operatorname{Id}(G)-\operatorname{Id}(G-e)=0, \operatorname{Id}(G)-\operatorname{Id}(G-e)= \pm 1$ or $\operatorname{Id}(G)-I d(G-e)= \pm 2$ exist.

- The case $r \geqslant 2$

Now the differences $I d_{r}(G)-I d_{r}(G-e)$ and $I d_{r}(G-e)-I d_{r}(G)$ can be large, and we obtain results which slightly vary with $r$.

Theorem 98 Let $k \geqslant 2$ be an arbitrary integer.

(a1) Let $r \geqslant 2$. There exist two $r$-twin-free graphs, $G$ and $G-e$, with $(r+1) k+r\left\lceil\log _{2}(k+2)\right\rceil+2 r$ vertices, such that $I d_{r}(G) \geqslant k$ and $I d_{r}(G-e) \leqslant r\left\lceil\log _{2}(k+2)\right\rceil+r+3$.

(a2) Let $r \geqslant 5$. There exist two $r$-twin-free graphs, $G$ and $G-e$, with $(2 r-2) k+r\left\lceil\log _{2}(k+2)\right\rceil+r+3$ vertices, such that $I d_{r}(G) \geqslant k$ and $I d_{r}(G-e) \leqslant r\left\lceil\log _{2}(k+2)\right\rceil+r+1$.

(b1) There exist two 2-twin-free graphs, $G$ and $G-e$, with $3 k+2\left\lceil\log _{2}(k+2)\right\rceil+5$ vertices, such that $I d_{2}(G-e) \geqslant k$ and $I d_{2}(G) \leqslant 2\left\lceil\log _{2}(k+2)\right\rceil+5$.

(b2) Let $r \geqslant 3$. There exist two $r$-twin-free graphs, $G$ and $G-e$, with $(2 r-2) k+r\left\lceil\log _{2}(k+2)\right\rceil+r+2$ vertices, such that $I d_{r}(G-e) \geqslant k$ and $I d_{r}(G) \leqslant r\left\lceil\log _{2}(k+2)\right\rceil+r+1$.

Very close results can be obtained with pairs of connected graphs. Whether these inequalities can be substantially improved is an open problem.

Note that $k$ can be taken arbitrarily and is linked to the order $n$ of $G$ and $G-e$ by the relation $n=\left(c_{1} r+c_{2}\right) k+r\left\lceil\log _{2}(k+2)\right\rceil+\left(c_{3} r+c_{4}\right)$ where the integer quadruple $\left(c_{1}, c_{2}, c_{3}, c_{4}\right)$ takes different values in (a1), (a2), (b1) and (b2) above. This means, roughly speaking, that $k$ is a fraction, depending on $r$, of $n$; therefore, Th. 98 implies that, given $r \geqslant 2$, there is an infinite sequence of graphs $G$ of order $n$ and two positive constants $\alpha$ and $\beta$ such that $I d_{r}(G) \geqslant \alpha n$ and, after deletion of a suitable edge $e, I d_{r}(G-e) \leqslant \beta \log _{2} n$ (or the other way round: $I d_{r}(G) \leqslant \beta \log _{2} n$ and $\left.I d_{r}(G-e) \geqslant \alpha n\right)$. We can see that adding or deleting one edge can lead to quite a drastic difference for the identification numbers. On this topic, see also [90] for a probabilistic approach.

\subsubsection{Identifying Codes: Adding or Deleting one Vertex or More}

- The case $r=1$

If $S \subset V$, we denote by $G-S$ the graph obtained from $G$ by deleting the vertices of $S$.

Theorem 99 ([83]) If $G$ and $G-S$ are 1-twin-free, then $\operatorname{Id}(G)-\operatorname{Id}(G-S) \leqslant|S|$. In particular, if $v \in V$, we have $\operatorname{Id}(G)-\operatorname{Id}(G-v) \leqslant 1$; moreover, the two inequalities are tight.

Theorem 100 ([151]) Let $n$ be the order of $G, S \subset V$ and $v \in V$.

(a) If $n \geqslant 2^{|S|-1}$, then $\operatorname{Id}(G-S)-I d(G) \leqslant n-2|S|-\left\lfloor\frac{n-|S|}{\left.2^{|S|}\right\rfloor}\right.$; the inequality is tight for $n$ sufficiently large with respect to $|S|$.

(b) We have $\operatorname{Id}(G-v)-I d(G) \leqslant \frac{n}{2}-\varepsilon$, with $\varepsilon=2$ if $n \in\{2,4,5,6,8\}, \varepsilon=1$ otherwise; the inequality is tight.

(c) If $G$ is bipartite, then $\operatorname{Id}(G-v)-I d(G) \leqslant \frac{n-\log _{2}\left(n-\log _{2} n\right)}{2}-1$; the inequality is tight. 
So, if one deletes a vertex, the identification number cannot drop by more than one, and can increase by a quantity close to $n / 2$.

- The case $r \geqslant 2$

Now both $I d_{r}(G-v)-I d_{r}(G)$ and $I d_{r}(G)-I d_{r}(G-v)$ can be large: the difference $I d_{r}(G-v)-I d_{r}(G)$ can increase to approximately $\frac{n}{4}$ (for even $r$ ) and $\frac{n(3 r-1)}{12 r}$ (for odd $r$ ), or $\frac{n(2 r-2)}{2 r+1}$, according to wether we want the graphs to be connected or not, and $I d_{r}(G)-I d_{r}(G-v)$ to approximately $\frac{n(r-1)}{r}$.

Theorem 101 ([36]) Let $n$ be the order of $G$ and $v \in V$.

(a) Let $r \geqslant 2$ be even. There exist two connected graphs, $G$ and $G-v$, such that $I d_{r}(G-v)-$ $I d_{r}(G) \geqslant \frac{n}{4}-(r+1)$.

(b) Let $r \geqslant 3$ be odd. There exist two connected graphs, $G$ and $G-v$, such that $I d_{r}(G-v)-$ $I d_{r}(G) \geqslant \frac{n(3 r-1)}{12 r}-r$. $2 r$.

(c) Let $r \geqslant 2$. There exist two graphs, $G$ and $G-v$, such that $I d_{r}(G-v)-I d_{r}(G) \geqslant \frac{(n-1)(2 r-2)}{2 r+1}-$

Theorem $102([36])$ Let $n$ be the order of $G$ and $v \in V$. Then there exist two connected graphs, $G$ and $G-v$, such that $I d_{r}(G)-I d_{r}(G-v) \geqslant \frac{(n-3 r-1)(r-1)+1}{r}$.

Whether all these inequalities can be substantially improved is an open problem.

Figure 6.16 illustrates Th. 102 for $n=17, r=3$.
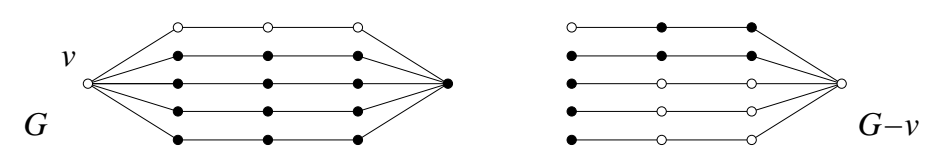

Fig. 6.16 In each graph, the black vertices form an optimal 3-Id code, and $I d_{3}(G)-I d_{3}(G-v)=13-8=5$.

\subsubsection{Criticality Concepts}

In this section, $r=1$. Let $\pi$ stand for $L D=L D_{1}$ or $I d=I d_{1}$. A graph $G=(V, E)$ is said to be $\pi^{+}$-edge removal critical, or $\pi^{+}$-ER-critical, if $\pi(G-e)>\pi(G)$ for all $e \in E$, and $\pi^{-}$-ER-critical if $\pi(G-e)<\pi(G)$ for all $e \in E$. Similarly, one can define $\pi^{+}$-vertex removal critical graphs and $\pi^{-}$-vertex removal critical graphs.

For instance, it is possible to characterize all graphs that are $\mathrm{LD}^{+}$-ER-critical. Let $H=\left(V_{1} \cup\right.$ $\left.V_{2}, E\right)$ be a connected bipartite graph such that: for every $w \in V_{2}$ and for every nonempty subset $V_{1}^{\prime} \subseteq N(w)$ there exists a unique $w^{\prime} \in V_{2}$ such that $N\left(w^{\prime}\right)=V_{1}^{\prime}$. Let $\mathcal{H}$ be the set of all such graphs, see Fig. 6.17 for an example.

Fig. 6.17 Two graphs belonging to $\mathcal{H}[21]$.

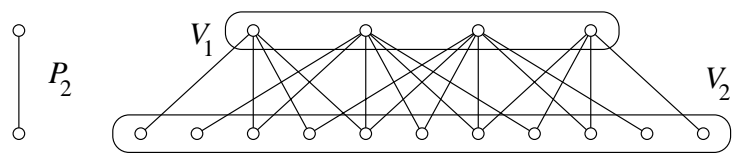


Theorem 103 ([21]) A nontrivial connected graph $G$ is $L D^{+}$-ER-critical if and only if $G \in \mathcal{H}$.

A nonempty graph is $L D^{+}-E R$-critical if and only if it is the disjoint union of independent sets and of graphs in $\mathcal{H}$.

For more results, see, e.g., [92] for graphs which are critical for identification, or [62] and [63] for locating-domination.

In Sect. 6.6.1, we mostly showed what a twin-free graph can become when we delete vertices; so far, in Sect. 6.6.2 we studied what the identification and locating-domination numbers can become when adding or deleting vertices or edges; but we can also be interested in what an existing (optimal) code becomes when edges or vertices are deleted or added-this leads naturally to the notion of robustness: an $r$-Id code $C$ is $t$-edge-robust [111] in $G$ if $C$ remains $r$-Id in all the graphs obtained from $G$ by adding or deleting edges, with a total amount of additions and deletions at most $t$; see also, among others, [139], [114]. Different definitions exist for $t$-vertex-robust codes, see [159], [111].

\subsection{Number of Optimal Codes}

In this section, we are interested in graphs with many optimal codes - or only one.

In order to obtain a large number of optimal 1-Id codes, Honkala, Hudry \& Lobstein [110] considered in 2015 the binary 3 -cube and used it to build a graph with $8 k+1$ vertices, $k \geqslant 1$, admitting at least $56^{k}+32 k \times 56^{k-1}$ optimal 1-Id codes, of size $4 k$, then they built graphs of higher orders and numbers of optimal codes, until they obtained the following result.

Theorem 104 ([110]) There exist infinitely many connected graphs of order $n$ admitting approximately $2^{0.770 \times n}$ different optimal 1-identifying codes.

For $r>1$, they used trees admitting many optimal codes, and combined them.

Theorem 105 ([110]) Let $r \geqslant 1$ be an integer and $\varepsilon>0$ be a real. There exist infinitely many connected graphs of order $n$ admitting $2^{\left(\frac{1+\log _{2} 5}{2}-\varepsilon\right) n}$ different optimal $r$-identifying codes.

Note that $\frac{1+\log _{2} 5}{2} \approx 0.664$. An obvious upper bound is $\left(\begin{array}{c}n \\ \left\lceil\frac{n}{2}\right\rceil\end{array}\right)$, which, using Stirling's formula, can be approximated above by $2^{n-\frac{1}{2} \log _{2} n}$; to reduce the gap between these lower and upper bounds remains an open problem.

In [95] there is the description of graphs $G$ where every $k$-set of vertices is a 1 -Id code, but the codes are not necessarily optimal, that is, $k$ may be different from $I_{1}(G)$. See a small example in Fig. 6.18(a).

On the other hand, a characterization of trees admitting a unique optimal 1-LD code is given in [19], see examples of such trees in Fig. 6.18(b).

For complexity issues related to the uniqueness of optimal codes, see Sect. 6.8.2.

\subsection{Complexity}

\subsubsection{How Hard is it to Find Optimal Codes?}

For any integer $r \geqslant 1$, we consider the following two decision problems. 
(a)

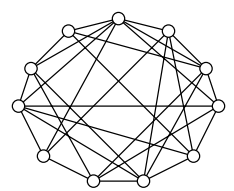

(b)

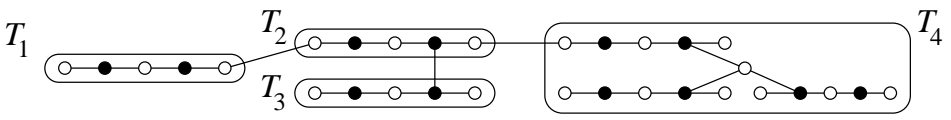

Fig. 6.18 (a) A graph where all sets with 7 vertices are 1-identifying codes (not optimal). (b) Codewords are in black. Each code inside $T_{1}, T_{2}, T_{3}, T_{4}, T_{1} \cup T_{2}, T_{2} \cup T_{3}, T_{2} \cup T_{4}, T_{1} \cup T_{2} \cup T_{3}, T_{1} \cup T_{2} \cup T_{4}, T_{2} \cup T_{3} \cup T_{4}$, $T_{1} \cup T_{2} \cup T_{3} \cup T_{4}$ is a unique optimal 1-locating-dominating code.

\section{$r$-LD CODE}

Instance: A graph $G=(V, E)$ and an integer $k \leqslant|V|$.

Question: Does $G$ admit an $r$-locatingdominating code of size at most $k$ ?

\section{$r$-Id CODE}

Instance: A graph $G=(V, E)$ and an integer $k \leqslant|V|$.

Question: Does $G$ admit an $r$-identifying code of size at most $k$ ?

Theorem 106 ([60]) The problem 1-LD CODE is NP-complete.

Proof. We simplify the 1987 proof by Colbourn, Slater \& Stewart in [60]. We take an arbitrary instance of 3-SAT, that is, a set $X=\left\{x_{1}, \ldots, x_{n}\right\}$ of variables and a set $\mathcal{C}=\left\{c_{1}, \ldots, c_{m}\right\}$ of clauses of size 3. For each variable $x$, we construct the 5-vertex graph $G_{x}=\left(V_{x}, E_{x}\right)$ given in Fig. 6.19(a), in which additional edges may be incident only with $x$ or $\bar{x}$.

(a)

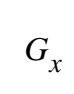

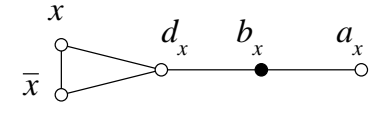

(b)

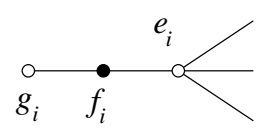

$H_{i}$

Fig. 6.19 Black vertices will be part of a LD code. (a) The graph $G_{x}$. (b) The graph $H_{i}$.

For each clause $c_{i}=\left\{\ell_{i, 1}, \ell_{i, 2}, \ell_{i, 3}\right\}$, we construct the 3 -vertex graph $H_{i}=\left(W_{i}, F_{i}\right)$ given by Fig. 6.19(b), where the 3 edges incident with $e_{i}$ and not with $f_{i}$, are incident with $\ell_{i, 1}, \ell_{i, 2}$, and $\ell_{i, 3}$. We claim that the resulting graph $G$, whose order $5 n+3 m$ is polynomial with respect to the size of the instance of 3-SAT, admits a LD code of size $k=2 n+m$ if and only if the set of clauses $\mathcal{C}$ is satisfiable.

(a) If $\mathcal{C}$ is satisfiable, take as codewords the $n$ vertices $b_{x}$, the $m$ vertices $f_{i}, 1 \leqslant i \leqslant m$, then for each variable $x \in X$, whichever of $x$ and $\bar{x}$ is true. This code, of size $k$, is locating-dominating, in particular because $e_{i}$ and $g_{i}$ are separated by at least one vertex corresponding to a true literal, i.e., are separated by a codeword.

(b) Let $C$ be a LD code in $G$, of size $k$. Obviously, $\left|C \cap V_{x}\right| \geqslant 2$ and $\left|C \cap W_{i}\right| \geqslant 1$; therefore, thanks to the choice of $k$, we have $\left|C \cap V_{x}\right|=2$ and $\left|C \cap W_{i}\right|=1$. Also, because $g_{i}$ must be dominated by $C$, we have $\left|C \cap\left\{g_{i}, f_{i}\right\}\right|=1$ and none of the vertices $e_{i}$ is in $C$. This implies that $|C \cap\{x, \bar{x}\}|=1$, because if $C \cap\{x, \bar{x}\}=\emptyset$, then the non-codewords $x$ and $\bar{x}$ are not separated by $C$ (the vertex $d_{x}$ cannot do it), and if $|C \cap\{x, \bar{x}\}|=2$, then $b_{x}$ and $a_{x}$ are not dominated by $C$. So we can define a valid truth assignment of the variables by setting $x$ true if and only if $x \in C$. 
(i) If $C \cap\left\{g_{i}, f_{i}\right\}=\left\{g_{i}\right\}$, then $e_{i}$ must be dominated by a codeword of type $x$ or $\bar{x}$; (ii) the same is true if $C \cap\left\{g_{i}, f_{i}\right\}=\left\{f_{i}\right\}$, because $g_{i}$ and $e_{i}$ must be separated by the code. This shows that every clause $c_{i}$ is satisfied by this assignment.

The same result for $r \geqslant 2$ was stated in [26] in 1995. We indicate the 2003 reference [40], which gives a proof.

Theorem 107 (a) ([26]), ([40]) For any $r>1$, the problem $r$-LD CODE is NP-complete.

(b) ([57] for $r=1)$, ([40]) For any $r \geqslant 1$, the problem $r$-Id CODE is NP-complete.

This means that, given $G$ and $r$, determining $L D_{r}(G)$ or $I d_{r}(G)$, or finding an optimal $r$-LD or $r$-Id code, is NP-hard.

Note that Th. 107 holds even if the instances are restricted to bipartite graphs; it also exists for bipartite oriented graphs [39]. For more on complexity, see [4], [6] for identification in planar graphs with arbitrarily high girth or low maximal degree, or [87] for interval and permutation graphs; Table 1 in [80] extends Table 1.8 in [79] and summarizes what was known around 2015 about the complexity of certain classes of graphs, for LD and Id codes, as well as dominating codes, for comparison.

See [118], [119] for identification in the binary $n$-cube, and [121] for problems closely related, such as the search for an $r$-Id code containing a prescribed vertex subset. For concepts close to locating-domination and identification that will be surveyed in Sect. 6.9: complexity results on $(r, \leqslant \ell)$-identifying codes are given in [6], on watching systems in [7], on open neighborhood locatingdominating codes in [164], on metric bases in [93, p. 204]; usually, the decision problems are NPcomplete, even for some restricted classes of graphs, but linear for trees (when $r=1$ ). The problem 1-LD CODE is also linear for series parallel graphs [60], and so is 1-Id CODE for block graphs [1]. For approximability issues, see, e.g., [80] (where Table 2 gives a state of play) or [87].

Complexity can bring what may look like surprises [79]: there are classes of graphs in which 1LD CODE is polynomial and 1-Id CODE is NP-complete, and, if we define the decision problem 1-DOM CODE similarly to 1-LD CODE or 1-Id CODE, there are even classes of graphs in which 1-LD CODE and 1-Id CODE are polynomial, whereas 1-DOM CODE is NP-complete.

\subsubsection{Uniqueness of Optimal Codes}

We consider the following decision problems, stated for fixed $r \geqslant 1$.

\begin{tabular}{|l|}
\hline UNIQUE OPTIMAL $r$-LD CODE \\
Instance: A graph $G=(V, E)$. \\
Question: Does $G$ admit a unique optimal $r$ - \\
locating-dominating code?
\end{tabular}

UNIQUE OPTIMAL $r$-Id CODE
Instance: A graph $G=(V, E)$.
Question: Does $G$ admit a unique optimal $r$ -
identifying code?

In [123], it is proved, among other results, that for every $r \geqslant 1$, these two problems are NP-hard and belong to the class $L^{N P}$ (also denoted by $P^{N P[O(\log n)]}$ or $\Theta_{2}$ ), which contains the decision problems which can be solved by applying, with a number of calls which is logarithmic with respect to the size of the instance, a subprogram able to solve an appropriate problem in NP. 


\subsection{Related Concepts, Generalizations}

- One of the first possible generalizations is to consider that more than one vertex may need to be retrieved by the code, and usually one puts a limit $\ell$ on the number of these vertices. Therefore, an $(r, \leqslant \ell)$-locating-dominating code $C$ in a graph $G=(V, E)$ is an $r$-dominating code such that for all $X \subseteq V \backslash C, Y \subseteq V \backslash C$, such that $X \neq Y,|X| \leqslant \ell,|Y| \leqslant \ell$, we have

$$
\bigcup_{u \in X} N_{r}(u) \cap C \neq \bigcup_{v \in Y} N_{r}(v) \cap C .
$$

One can similarly define $(r, \leqslant \ell)$-identifying codes. Both generalizations, and other similar ones, have been studied mostly in the $n$-cube and in the grids; we refer to, e.g., [134], [137], [112], [116], [97], [73], [70], [150], [86].

- Next, considering that using $r$-LD or $r$-Id codes amounts to using $r$-neighborhoods centered at the codewords, one can think of changing the pattern surrounding a codeword.

One choice of pattern is, given $G=(V, E)$ and $r \geqslant 1$, to consider that every codeword $c \in C$ can check a connected subset of $N_{r}[c]$, instead of the whole set $N_{r}[c]$ for an $r$-identifying code, and that several codewords can be on the same vertex. This leads to the notion of watching systems [7], which are more complex but can be very efficient: consider for instance the star $K_{1, n-1}$, which requires as many as $n-1$ codewords for 1 -identification and even for 1-locating-domination, and as few as $\left\lceil\log _{2}(n+1)\right\rceil$ if one puts suitable watchers at the centre of the star. See also [9], [8]. When a watching system has at most one codeword on each vertex, we talk of choice identification [27].

Other patterns for different graphs can be thought of: cycles, paths (including paths of even order in the infinite path, as opposed to odd paths which are closed neighborhoods around their centres), squares of even side in $\mathbb{Z} \times \mathbb{Z}$, as opposed to odd squares, which are closed neighborhoods in the king grid, etc. See, e.g., [120], [162], [85].

- A slight modification in the definition of a 1-identifying code leads to open neighborhood locating-dominating codes (OLD codes): an OLD code $C$ is such that for every two vertices $u$, $v$ of $V(G)$, the sets $N(u) \cap C$ and $N(v) \cap C$ are nonempty and different. This was introduced in [115] (for $r \geqslant 1$ and for identification of sets of vertices) in the binary $n$-cube, then extended to all graphs in [164] (for $r=1$ ). This describes a situation where an intruder at a vertex $v$ can prevent the detection device at $v$ from signalling the intrusion, that is, $v$ can only check $N(v)$. In most of our models, we assumed that a codeword correctly sent a 1 if it detected something in its neighborhood, a 0 otherwise. The definition of OLD codes is one of several definitions for so-called fault-tolerant codes, where different scenarii are considered for the alarms given by the codewords.

If $O L D(G)$ denotes the minimum size of an OLD code in $G$, the only graphs with $O L D(G)=$ $|V(G)|$ are the three graphs given by Fig. 6.20 and the only graphs with $O L D(G)=2$ are the complete graphs $K_{2}$ and $K_{3}$ [51]. Another representative result is that the minimum density of an OLD code in the triangular grid is $4 / 13 \approx 0.3077$ [136], to be compared with 0.25 for a 1-identifying code, see Th. 32. For more on this topic, see also, e.g., [165], [176], [163].

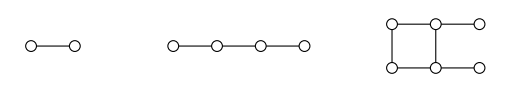

Fig. 6.20 The three graphs $G=(V, E)$ with $O L D(G)=|V|$. 
One can also mention here the liar's problem, when the vertices of $C$ can tell precisely where the malfunctioning vertex $v$ is if it belongs to their closed neighborhoods, so a dominating code would be enough, except that any one codeword in the closed neighborhood of $v$ can lie, that is, either misidentify any vertex in its closed neighborhood as $v$, or fail to report any vertex. For instance, three codewords are necessary and sufficient in the complete graph $K_{n}$ with $n \geqslant 3$. See [173] or [161].

- Another related definition is the following: a code $C$ is strongly $r$-identifying [115] if for all $v_{1} \in V, v_{2} \in V, v_{1} \neq v_{2}$, the sets $\left\{N_{r}\left[v_{1}\right] \cap C, N_{r}\left(v_{1}\right) \cap C\right\}$ and $\left\{N_{r}\left[v_{2}\right] \cap C, N_{r}\left(v_{2}\right) \cap C\right\}$ are disjoint; this can be extended to codes identifying more than one vertex. See [109], where the best density for a strongly 1 -Id code in the triangular grid is proved to be $6 / 19 \approx 0.3158$, or [138], [141].

- Using identifying codes in search of one defective vertex can be seen as asking to the codewords, all at one time: "Is there a defective vertex in your closed neighborhood?" Adaptive identification consists in asking the queries one by one, taking into account the previous answers. So, after the first yes answer is obtained in one neighborhood, we dichotomize this neighborhood with other neighborhoods (= new queries) so as to minimize the maximum number of queries [12], [127], [135].

- The notions of 1-LD and 1-Id codes can be modified by adding the condition that the code, instead of being dominating, must be total dominating: a total dominating code $C$ of a graph $G=(V, E)$ is a locating-total dominating code if for every pair of distinct vertices $u$ and $v$ in $V \backslash C$, one has $N(u) \cap C \neq N(v) \cap C$, and $C$ is a differentiating-total dominating code, or an identifyingtotal dominating code, if for every pair of distinct vertices $u$ and $v$ in $V, N[u] \cap C \neq N[v] \cap C$. See, e.g., [103], [49], [53], [22], [105], [146], [129].

- An older way of locating vertices is the following: given a graph $G=(V, E)$ and a code $C=$ $\left\{c_{1}, c_{2}, \ldots, c_{k}\right\}$, the $C$-location of a vertex $v \in V$ is the distance vector $\left(d\left(v, c_{1}\right), d\left(v, c_{2}\right), \ldots, d\left(v, c_{k}\right)\right)$. If no two vertices have the same $C$-location, then $C$ is said to be resolving, as introduced in [101], or locating, as introduced independently in [167], around 1975. An optimal resolving code is called a metric basis and its size is the metric dimension of the graph. For example, the only graphs with metric dimension 1 are the paths, for which each endvertex constitutes a resolving code. See also [24], [87], or [100]; see [104] for metric-locating-domination (where the code must also be 1-dominating), and [48] for the related concepts of distance-location and external distance-location.

Moreover, if we have a proper vertex coloring $c$ with $k$ colors, and if $\Pi=\left(C_{1}, C_{2}, \ldots, C_{k}\right)$ is the resulting partition of $V$, we can define, for every $v \in V$, the color code of $v$, that is, the distance vector $\left(d\left(v, C_{1}\right), d\left(v, C_{2}\right), \ldots, d\left(v, C_{k}\right)\right)$. If distinct vertices have distinct color codes, then $c$ is called a locating-coloring. The locating-chromatic number of $G$ is the minimum number of colors in a locating-coloring. See, e.g., [46], [47], [11]. Other more or less distant concepts using colorings exist, such as locally identifying colorings [69], and many more, see, e.g., [181], [148], [180].

- For the model of information retrieval in associative memories, see, e.g., [179], [132], [140].

- The d-identifying codes [177] generalize Id codes by endowing every vertex with a positive integer cost, which will give a global cost to the code that may be different from its size, and with a positive integer weight which, together with $d$, allows and measures a degree of uncertainty in the identification.

- Another parameter, the propagation time, can be considered; in this model, a fault at one vertex $v$ in a weighted digraph spreads along the arcs and reaches any out-neighbor $w$ in a time equal to the weight of the arc $v w$, then spreads from $w$, and so on. See, e.g., [158], [14].

- Other definitions: paired-LD or Id codes (the code must be paired-dominating), co-isolated LD or Id codes (the code $C$ is such that there exists at least one isolated vertex in $\langle V-C\rangle$ ), independent LD or Id codes, connected codes, weak r-codes and light r-codes, self-LD or Id codes, solid-LD codes. 
It is also possible to generalize these concepts to hypergraphs, and of course, some of these definitions can be combined, e.g., connected vertex-robust identifying codes [78].

\subsection{Concluding Remarks, Open Problems, Conjectures}

We have tried to give an overview of the major results on locating-domination and on identification. We apologize in advance if your favorite variation or result is omitted. We simply refer people interested to know more about these concepts to [142] for more literature on the topic; they can search for key-words there, or on the web for even more references.

We conclude with two seemingly paradoxical remarks partly collected from [160]:

(a) For some graphs, increasing $r$, i.e., increasing the power of the codewords, can require more codewords; this was first noticed in [26], where a tree $T$ is given, such that $L D_{2}(T)=L D_{3}(T)=6$ and $L D_{4}(T)=5$, while $L D_{7}(T)>6$. Paths provide similar cases for identification; for instance, if $k \geqslant 1$, then by Th. 44 we have $I d_{1}\left(P_{10 k+5}\right)=5 k+3$, whereas by Th. 49 (a) we have $I d_{2}\left(P_{10 k+5}\right)=$ $5 k+4$. In the case of the binary $n$-cube, we have $I d_{1}\left(\mathbb{F}^{6}\right)=19, I d_{2}\left(\mathbb{F}^{6}\right)=8, I d_{3}\left(\mathbb{F}^{6}\right)=7$, $I d_{4}\left(\mathbb{F}^{6}\right)=18, I d_{5}\left(\mathbb{F}^{6}\right)=63$.

This paradox can be understood when $r$ becomes relatively large with respect to the graph: if the $r$-neighborhoods grow too much, they cannot separate anything anymore; the extremal case is when $r$ reaches the diameter of the graph, where $n-1$ codewords are needed for an $r$-LD code, and no $r$-Id code exists. But this is not the case for the example of the path $P_{10 k+5}$ above.

(b) If we take a path or a cycle and make it longer, it may need fewer codewords; for instance, for $k \geqslant 1$, one has $I d_{2}\left(C_{10 k+6}\right)=I d_{2}\left(C_{10 k+5}\right)-2$.

The discussion following Th. 62 gives another example, which however can be explained by the fact that increasing the dimension $n$ of the cube makes $r$ not too large with respect to $n$ anymore.

Finally, we recapitulate the open problems and conjectures given throughout the text.

Open Problem 1 [2020] (see End of Section 6.3). For $r \geqslant 2$, most results on the costs of locating-domination vs domination are exact but valid for $n$ large. (a) Establish results for all $n$. (b) Reduce the gap between lower and upper bounds for $F_{L D, \gamma}(r, n)$.

Open Problem 2 [2011] (see Th. 87). For $r \geqslant 5$, find the exact value of $f_{r}\left(I d_{r}\right)$, knowing that it lies between $\left\lceil\log _{2}(2 r+4)\right\rceil$ and $r+1$.

Open Problem 3 [2007] (see End of Section 6.6.1.1). (a) Apart from $P_{5}$, do 2-terminal graphs exist? (b) There exist $r$-terminal graphs for $r \in\{3,4,5\}$, but are they in finite or infinite number?

Open Problem 4 [2013]. Improve the bounds in Ths. 98, 101 and 102.

Open Problem 5 [2015] (see Ths. 104 and 105). Reduce the gap between the lower and upper bounds on the number of different optimal identifying codes.

Conjecture 1 [1995] (see Th. 54). For any tree $T$ of order $n$, we have $L D_{r}(T)>\frac{n}{r+2}$.

Conjecture 2 [1999] (see the discussion following Th. 60). (a) We have $I d_{r_{1}+r_{2}}\left(\mathbb{F}^{n_{1}+n_{2}}\right) \leqslant$ $I d_{r_{1}}\left(\mathbb{F}^{n_{1}}\right) \times I d_{r_{2}}\left(\mathbb{F}^{n_{2}}\right)$. (b) We have $\operatorname{Id}\left(\mathbb{F}^{n+1}\right) \leqslant 2 \operatorname{Id}\left(\mathbb{F}^{n}\right)$.

Conjecture 3 [2010] (see the discussion after Th. 62). For a fixed $r>1$, there exists $n(r)$ such that for all $n \geqslant n(r)$, we have $I d_{r}\left(\mathbb{F}^{n}\right) \leqslant I d_{r}\left(\mathbb{F}^{n+1}\right)$.

Conjecture 4 [2012] (see the discussion following Th. 71). There exists a constant $c$ such that, for every nontrivial connected 1-twin-free graph $G$, with maximum degree $\Delta$, we have $\operatorname{Id}_{1}(G) \leqslant$ $n-\frac{n}{\Delta}+c$.

Conjecture 5 [2009] (see Th. 79). The value $F_{r, n}(\delta)$ is close, possibly equal, to $\left\lfloor\frac{n-2}{r}\right\rfloor$. 


\section{References}

1. G. R. Argiroffo, S. M. Bianchi, Y. P. Lucarini and A. K. Wagler, A linear-time algorithm for the identifying code problem on block graphs. Electron. Notes Discrete Math. 62 (2017), 249-254.

2. G. R. Argiroffo, S. M. Bianchi, Y. P. Lucarini and A. K. Wagler, Polyhedra associated with identifying codes in graphs. Discrete Appl. Math. 245 (2018), 16-27.

3. D. Auger, Induced paths in twin-free graphs. Electron. J. Combin. 15(1) (2008), N17.

4. D. Auger, Minimal identifying codes in trees and planar graphs with large girth. European J. Combin. 31 (2010), 1372-1384.

5. D. Auger, I. Charon, I. Honkala, O. Hudry and A. Lobstein, Edge number, minimum degree, maximum independent set, radius and diameter in twin-free graphs. Adv. Math. Commun. 3(1) (2009), 97-114. Erratum 3(4) (2009), 429-430.

6. D. Auger, I. Charon, O. Hudry and A. Lobstein, Complexity results for identifying codes in planar graphs. Int. Trans. Oper. Res. 17 (2010), 691-710.

7. D. Auger, I. Charon, O. Hudry and A. Lobstein, Watching systems in graphs: an extension of identifying codes. Discrete Appl. Math. 161 (2013), 1674-1685.

8. D. Auger, I. Charon, O. Hudry and A. Lobstein, Maximum size of a minimum watching system and the graphs achieving the bound. Discrete Appl. Math. 164 (2014), 20-33.

9. D. Auger and I. Honkala, Watching systems in the king grid. Graphs Combin. 29 (2013), 333-347.

10. C. Balbuena, F. Foucaud and A. Hansberg, Locating-dominating sets and identifying codes in graphs of girth at least 5. Electron. J. Combin. 22(2), (2015) P2.15.

11. A. Behtoei and M. Anbarloei, A bound for the locating chromatic number of trees. Transactions on Combinatorics 4(1) (2015), 31-41.

12. Y. Ben-Haim, S. Gravier, A. Lobstein and J. Moncel, Adaptive identification in torii in the king lattice. Electron. J. Combin. 18(1) (2011), P116.

13. Y. Ben-Haim and S. Litsyn, Exact minimum density of codes identifying vertices in the square grid. SIAM J. Discrete Math. 19 (2005), 69-82.

14. T. Y. Berger-Wolf, W. E. Hart and J. Saia, Discrete sensor placement problems in distribution networks. Math. Comput. Modelling 42 (2005), 1385-1396.

15. N. Bertrand, Codes identifiants et codes localisateurs-dominateurs sur certains graphes. Mémoire de stage de maîtrise. ENST, Paris, France, (2001), 28 pages.

16. N. Bertrand, I. Charon, O. Hudry and A. Lobstein, Identifying and locating-dominating codes on chains and cycles. European J. Combin. 25 (2004), 969-987.

17. N. Bertrand, I. Charon, O. Hudry and A. Lobstein, 1-identifying codes on trees. Australas. J. Combin. 31 (2005), 21-35.

18. U. Blass, I. Honkala and S. Litsyn, On the size of identifying codes. Lecture Notes in Comput. Sci. 1719 (1999), 142-147.

19. M. Blidia, M. Chellali, R. Lounes and F. Maffray, Characterizations of trees with unique minimum locating-dominating sets. J. Combin. Math. Combin. Comput. 76 (2011), 225-232.

20. M. Blidia, M. Chellali, F. Maffray, J. Moncel and A. Semri, Locating-domination and identifying codes in trees. Australas. J. Combin. 39 (2007), 219-232.

21. M. Blidia and W. Dali, A characterization of locating-domination edge critical graphs. Australas. J. Combin. 44 (2009), 297-300.

22. M. Bouznif, Algorithmes génériques en temps constant pour la résolution de problèmes combinatoires dans la classe des rotagraphes et fasciagraphes. Application aux codes identifiants, dominantlocalisateurs et total-dominant-localisateurs. Thèse de Doctorat. Université de Grenoble, France, (2012), 131 pages.

23. M. Bouznif, F. Havet, M. Preissmann, Minimum-density identifying codes in square grids. Lecture Notes in Comput. Sci. 9778 (2016), 77-88.

24. J. Cáceres, D. Garijo, M. L. Puertas and C. Seara, On the determining number and the metric dimension of graphs. Electron. J. Combin. 17(1) (2010), R63.

25. J. Cáceres, C. Hernando, M. Mora, I. M. Pelayo and M. L. Puertas, Locating-dominating codes: bounds and extremal cardinalities. Appl. Math. Comput. 220 (2013), 38-45.

26. D. I. Carson, On generalized location-domination. In Graph Theory, Combinatorics, and Applications: Proceedings of the 7th Quadrennial International Conference on the Theory and Applications of Graphs, Wiley, 1 (1995), 161-179. 
27. T. P. Chang and L. D. Tong, Choice identification of a graph. Discrete Appl. Math. 167 (2014), 61-71.

28. E. Charbit, I. Charon, G. Cohen, O. Hudry and A. Lobstein, Discriminating codes in bipartite graphs: bounds, extremal cardinalities, complexity. Adv. Math. Commun. 4(2) (2008), 403-420.

29. I. Charon, G. Cohen, O. Hudry and A. Lobstein, Links between discriminating and identifying codes in the binary Hamming space. Lecture Notes in Computer Science, No. 4851, pp. 267-270, Springer-Verlag, 2007.

30. I. Charon, G. Cohen, O. Hudry and A. Lobstein, Discriminating codes in (bipartite) planar graphs. European J. Combin. 29 (2008), 1353-1364.

31. I. Charon, G. Cohen, O. Hudry and A. Lobstein, New identifying codes in the binary Hamming space. European J. Combin. 31 (2010), 491-501. See also: perso.telecom-paristech.fr/ hudry/newIdentifyingNcube.html

32. I. Charon, S. Gravier, O. Hudry, A. Lobstein, M. Mollard and J. Moncel, A linear algorithm for minimum 1-identifying codes in oriented trees. Discrete Appl. Math. 154 (2006), 1246-1253.

33. I. Charon, I. Honkala, O. Hudry and A. Lobstein, General bounds for identifying codes in some infinite regular graphs. Electron. J. Combin. 8(1) (2001), R39.

34. I. Charon, I. Honkala, O. Hudry and A. Lobstein, The minimum density of an identifying code in the king lattice. Discrete Math. 276 (2004), 95-109.

35. I. Charon, I. Honkala, O. Hudry and A. Lobstein, Structural properties of twin-free graphs. Electron. J. Combin. 14(1) (2007), R16.

36. I. Charon, I. Honkala, O. Hudry and A. Lobstein, Minimum sizes of identifying codes in graphs differing by one vertex. Cryptogr. Commun. 5 (2013), 119-136.

37. I. Charon, I. Honkala, O. Hudry and A. Lobstein, Minimum sizes of identifying codes in graphs differing by one edge. Cryptogr. Commun. 6 (2014), 157-170.

38. I. Charon, O. Hudry and A. Lobstein, Identifying codes with small radius in some infinite regular graphs. Electron. J. Combin. 9(1) (2002), R11.

39. I. Charon, O. Hudry and A. Lobstein, Identifying and locating-dominating codes: NP-completeness results for directed graphs. IEEE Trans. Inform. Theory IT-48 (2002), 2192-2200.

40. I. Charon, O. Hudry and A. Lobstein, Minimizing the size of an identifying or locating-dominating code in a graph is NP-hard. Theoret. Comput. Sci. 290 (2003), 2109-2120.

41. I. Charon, O. Hudry and A. Lobstein, Possible cardinalities for identifying codes in graphs. Australas. J. Combin. 32 (2005), 177-195.

42. I. Charon, O. Hudry and A. Lobstein, Possible cardinalities for locating-dominating codes in graphs. Australas. J. Combin. 34 (2006), 23-32.

43. I. Charon, O. Hudry and A. Lobstein, Extremal cardinalities for identifying and locating-dominating codes. Discrete Math., 307 (2007), 356-366.

44. I. Charon, O. Hudry and A. Lobstein, Extremal values for identification, domination and maximum cliques in twin-free graphs. Ars Combin. 101 (2011), 161-185.

45. I. Charon, O. Hudry and A. Lobstein, Extremal values for the maximum degree in a twin-free graph. Ars Combin. 107 (2012), 257-274.

46. G. Chartrand, D. Erwin, M. A. Henning, P. J. Slater and P. Zhang, The locating-chromatic number of a graph. Bull. Inst. Combin. Appl. 36 (2002), 89-101.

47. G. Chartrand, D. Erwin, M. A. Henning, P. J. Slater and P. Zhang, Graphs of order $n$ with locatingchromatic number $n-1$. Discrete Math. 269 (2003), 65-79.

48. G. Chartrand, D. Erwin, P. J. Slater and P. Zhang, Distance-location numbers of graphs. Util. Math. 63 (2003), 65-79.

49. M. Chellali, On locating and differentiating-total domination in trees. Discuss. Math. Graph Theory 28 (2008), 383-392.

50. M. Chellali, M. Mimouni and P. J. Slater, On locating-domination in graphs. Discuss. Math. Graph Theory 30 (2010), 223-235.

51. M. Chellali, N. J. Rad, S. J. Seo and P. J. Slater, On open neighborhood locating-dominating in graphs. Electron. J. Graph Theory and Applications 2(2) (2014), 87-98.

52. C. Chen, C. Lu and Z. Miao, Identifying codes and locating-dominating sets on paths and cycles. Discrete Appl. Math. 159 (2011), 1540-1547.

53. X. Chen and M. Y. Sohn, Bounds on the locating-total domination number of a tree. Discrete Appl. Math. 159 (2011), 769-773. 
54. G. Cohen, S. Gravier, I. Honkala, A. Lobstein, M. Mollard, Ch. Payan and G. Zémor, Improved identifying codes for the grid. Electron. J. Combin. 6(1) (1999), Comments to R19.

55. G. Cohen, I. Honkala, S. Litsyn and A. Lobstein, Covering Codes, Elsevier, Amsterdam, 1997.

56. G. Cohen, I. Honkala, A. Lobstein and G. Zémor, Bounds for codes identifying vertices in the hexagonal grid. SIAM J. Discrete Math. 13 (2000), 492-504.

57. G. Cohen, I. Honkala, A. Lobstein and G. Zémor, On identifying codes. In Proceedings of DIMACS Workshop on Codes and Association Schemes '99, Piscataway, USA, Barg A. and Litsyn S. (eds), American Mathematical Society, 2001, 56 97-109.

58. G. Cohen, I. Honkala, A. Lobstein and G. Zémor, On codes identifying vertices in the two-dimensional square lattice with diagonals. IEEE Trans. Comput. 50 (2001), 174-176.

59. N. Cohen and F. Havet, On the minimum size of an identifying code over all orientations of a graph. Electron. J. Combin. 25(1) (2018), P1.49.

60. C. J. Colbourn, P. J. Slater and L. K. Stewart, Locating dominating sets in series parallel networks. Congr. Numer. 56 (1987), 135-162.

61. A. Cukierman and G. Yu, New bounds on the minimum density of an identifying code for the infinite hexagonal grid. Discrete Appl. Math. 161 (2013), 2910-2924.

62. W. Dali and M. Blidia, Criticality indices of locating-domination of paths and cycles. Util. Math. 94 (2014), 199-219.

63. W. Dali and M. Blidia, On locating and locating-total domination edge addition critical graphs. Util. Math. 94 (2014), 303-313.

64. M. Daniel, Codes identifiants. Mémoire pour le DEA ROCO. Université Joseph Fourier, Grenoble, France, (2003), 46 pages.

65. M. Daniel, S. Gravier and J. Moncel, Identifying codes in some subgraphs of the square lattice. Theoret. Comput. Sci. 319 (2004), 411-421.

66. R. Dantas, F. Havet and R. M. Sampaio, Identifying codes for infinite triangular grids with a finite number of rows. Discrete Math. 340 (2017), 1584-1597.

67. R. Dantas, F. Havet and R. M. Sampaio, Minimum density of identifying codes of king grids. Discrete Math. 341 (2018), 2708-2719.

68. R. C. Entringer and L. D. Gassman, Line-critical point determining and point distinguishing graphs. Discrete Math., 10 (1974), 43-55.

69. L. Esperet, S. Gravier, M. Montassier, P. Ochem and A. Parreau, Locally identifying coloring of graphs. Electron. J. Combin. 19(2) (2012), P40.

70. G. Exoo, V. Junnila and T. Laihonen, On location-domination of set of vertices in cycles and paths. Congr. Numer. 202 (2010), 97-112.

71. G. Exoo, V. Junnila and T. Laihonen, Locating-dominating codes in paths. Discrete Math. 311 (2011), $1863-1873$.

72. G. Exoo, V. Junnila and T. Laihonen, Locating-dominating codes in cycles. Australas. J. Combin. 49 (2011), 177-194.

73. G. Exoo, V. Junnila, T. Laihonen and S. Ranto, Locating vertices using codes. Congr. Numer. 191 (2008), 143-159.

74. G. Exoo, V. Junnila, T. Laihonen and S. Ranto, Upper bounds for binary identifying codes. Adv. in Appl. Math. 42 (2009), 277-289.

75. G. Exoo, V. Junnila, T. Laihonen and S. Ranto, Improved bounds on identifying codes in binary Hamming spaces. European J. Combin. 31 (2010), 813-827.

76. G. Exoo, T. Laihonen and S. Ranto, Improved upper bounds on binary identifying codes. IEEE Trans. Inform. Theory IT-53 (2007), 4255-4260.

77. G. Exoo, T. Laihonen and S. Ranto, New bounds on binary identifying codes. Discrete Appl. Math. 156 (2008), 2250-2263.

78. N. Fazlollahi, D. Starobinski and A. Trachtenberg, Connecting identifying codes and fundamental bounds. Proceedings of Information Theory and Applications Workshop ITA 2011, La Jolla, USA (2011), 403409.

79. F. Foucaud, Aspects combinatoires et algorithmiques des codes identifiants dans les graphes. Thèse de Doctorat, Université Bordeaux 1, France, (2012), 194 pages (in English).

80. F. Foucaud, Decision and approximation complexity for identifying codes and locating-dominating sets in restricted graph classes. J. Discrete Alg. 31 (2015), 48-68. 
81. F. Foucaud, S. Gravier, R. Naserasr, A. Parreau, and P. Valicov, Identifying codes in line graphs. J. Graph Theory $\mathbf{7 3}$ (2013), pp. 425-448.

82. F. Foucaud, E. Guerrini, M. Kovše, R. Naserasr, A. Parreau and P. Valicov, Classifying graphs with minimum identifying codes of size $n-1$, Abstracts of the 8th French Combinatorial Conference, Orsay, France, 151 (2010).

83. F. Foucaud, E. Guerrini, M. Kovše, R. Naserasr, A. Parreau and P. Valicov, Extremal graphs for the identifying code problem. European J. Combin. 32 (2011), 628-638.

84. F. Foucaud, R. Klasing, A. Kosowski and A. Raspaud, On the size of identifying codes in triangle-free graphs. Discrete Appl. Math. 160 (2012), 1532-1546.

85. F. Foucaud and M. Kovše, Identifying path covers in graphs, J. Discrete Alg. 23 (2013), 21-34.

86. F. Foucaud, T. Laihonen and A. Parreau, An improved lower bound for $(1, \leqslant 2)$-identifying codes in the king grid. Adv. Math. Commun. 8 (2014), 35-52.

87. F. Foucaud, G. B. Mertzios, R. Naserasr, A. Parreau and P. Valicov, Identification, location-domination and metric dimension on interval and permutation graphs: II. Algorithms and complexity, Algorithmica 78 (2017), 914-944.

88. F. Foucaud, R. Naserasr and A. Parreau, Characterizing extremal digraphs for identifying codes and extremal cases of Bondy's theorem on induced subsets. Graphs Combin. 29 (2013), 463-473.

89. F. Foucaud and G. Perarnau, Bounds for identifying codes in terms of degree parameters. Electron. J. Combin. 19(1) (2012), P32.

90. F. Foucaud, G. Perarnau and O. Serra, Random subgraphs make identification affordable. J. Comb. 8 (2017), 57-77.

91. M. Frances and A. Litman, On covering problems of codes. Theory Comput. Syst. 30(2) (1997), 113-119.

92. M. Frick, G. H. Fricke, C. M. Mynhardt and R. D. Skaggs, Critical graphs with respect to vertex identification. Util. Math. 76 (2008), 213-227.

93. M. R. Garey and D. S. Johnson, Computers and Intractability, a Guide to the Theory of NPCompleteness, Freeman, New York, 1979.

94. J. Gimbel, B. D. Van Gorden, M. Nicolescu, C. Umstead and N. Vaiana, Location with dominating sets. Congr. Numer. 151 (2001), 129-144.

95. S. Gravier, S. Janson, T. Laihonen and S. Ranto, Graphs where every $k$-subset of vertices is an identifying set. Discrete Math. Theor. Comput. Sci. 16 (2014), 73-88.

96. S. Gravier, R. Klasing and J. Moncel, Hardness results and approximation algorithms for identifying codes and locating-dominating codes in graphs. Algorithmic Oper. Res. 3 (2008), 43-50.

97. S. Gravier and J. Moncel, Construction of codes identifying sets of vertices. Electron. J. Combin. 12(1) (2005), R13.

98. S. Gravier and J. Moncel, On graphs having a $V \backslash\{x\}$ set as an identifying code. Discrete Math. 307 (2007), 432-434.

99. S. Gravier, J. Moncel and A. Semri, Identifying codes of cycles. European J. Combin. 27 (2006), 767-776.

100. A. Hakanen and T. Laihonen, On $\{\ell\}$-metric dimensions in graphs. Fund. Inform. 162 (2018), 143-160.

101. F. Harary and R. A. Melter, On the metric dimension of a graph. Ars Combin. 2 (1976), 191-195. Addendum 4 (1977), 318.

102. T. W. Haynes, S. T. Hedetniemi and P. J. Slater, Fundamentals of Domination in Graphs, Marcel Dekker, New York, 1998.

103. T. W. Haynes, M. A. Henning and J. Howard, Locating and total dominating sets in trees. Discrete Appl. Math. 154 (2006), 1293-1300

104. M. A. Henning and O. R. Oellermann, Metric-locating-dominating sets in graphs. Ars Combin. 73 (2004), $129-141$.

105. M. A. Henning and N. J. Rad, Locating-total domination in graphs. Discrete Appl. Math. 160 (2012), 1986-1993.

106. C. Hernando, M. Mora and I. M. Pelayo, Nordhaus-Gaddum bounds for locating domination. European J. Combin. 36 (2014), 1-6.

107. I. Honkala, An optimal locating-dominating set in the infinite triangular grid. Discrete Math. 306 (2006), 2670-2681.

108. I. Honkala, On $r$-locating-dominating sets in paths. European J. Combin. 30 (2009), 1022-1025.

109. I. Honkala, An optimal strongly identifying code in the infinite triangular grid. Electron. J. Combin. 17(1) (2010), R91. 
110. I. Honkala, O. Hudry and A. Lobstein, On the number of optimal identifying codes in a twin-free graph. Discrete Appl. Math. 180 (2015), 111-119.

111. I. Honkala, M. G. Karpovsky and L. B. Levitin, On robust and dynamic identifying codes. IEEE Trans. Inform. Theory IT-52 (2006), 599-612.

112. I. Honkala and T. Laihonen, On identifying codes in the triangular and square grids. SIAM J. Comput. 33 (2004), 304-312.

113. I. Honkala and T. Laihonen, On locating-dominating sets in infinite grids. European J. Combin. 27 (2006), 218-227.

114. I. Honkala and T. Laihonen, On identifying codes that are robust against edge changes. Inform. and Comput. 205 (2007), 1078-1095.

115. I. Honkala, T. Laihonen and S. Ranto, On strongly identifying codes. Discrete Math. 254 (2002), 191-205.

116. I. Honkala, T. Laihonen and S. Ranto, On locating-dominating codes in binary Hamming spaces. Discrete Math. Theor. Comput. Sci. 6 (2004), 265-282.

117. I. Honkala and A. Lobstein, On the density of identifying codes in the square lattice. J. Combin. Theory, Ser. B 85 (2002), 297-306.

118. I. Honkala and A. Lobstein, On identifying codes in binary Hamming spaces. J. Combin. Theory, Ser. A 99 (2002), 232-243.

119. I. Honkala and A. Lobstein, On the complexity of the identification problem in Hamming spaces. Acta Inform. 38 (2002), 839-845.

120. I. Honkala and A. Lobstein, On identification in $Z^{2}$ using translates of given patterns. J. UCS 9(10) (2003), 1204-1219.

121. O. Hudry and A. Lobstein, More results on the complexity of identifying problems in graphs. Theoret. Comput. Sci. 626 (2016), 1-12.

122. O. Hudry and A. Lobstein, Some results about a conjecture on identifying codes in complete suns. Int. Trans. Oper. Res. 26 (2019), 732-746.

123. O. Hudry and A. Lobstein, Unique (optimal) solutions: Complexity results for identifying and locatingdominating codes. Theoret. Comput. Sci. 767 (2019), 83-102.

124. O. Hudry and A. Lobstein, The compared costs of domination, location-domination and identification. Discussiones Mathematicae Graph Theory 40(1) (2020), 127-147.

125. S. Janson and T. Laihonen, On the size of identifying codes in binary hypercubes. J. Combin. Theory, Ser. A 116 (2009), 1087-1096.

126. M. Jiang, Periodicity of identifying codes in strips. Inform. Process. Lett. 135 (2018), 77-84.

127. V. Junnila, Adaptive identification of sets of vertices in graphs. Discrete Math. Theor. Comput. Sci. 14 (2012), 69-86.

128. V. Junnila, New lower bound for 2-identifying code in the square grid. Discrete Appl. Math. 161 (2013), 2042-2051.

129. V. Junnila, Optimal locating-total dominating sets in strips of height 3. Discuss. Math. Graph Theory 35 (2015), 447-462.

130. V. Junnila and T. Laihonen, Optimal identifying codes in cycles and paths. Graphs Combin. 28 (2012), $469-481$.

131. V. Junnila and T. Laihonen, Optimal lower bound for 2-identifying codes in the hexagonal grid. Electron. J. Combin. 19(2) (2012), P38.

132. V. Junnila and T. Laihonen, Codes for information retrieval with small uncertainty. IEEE Trans. Inform. Theory IT-60 (2014), 976-985.

133. V. Junnila, T. Laihonen and T. Lehtilä, On regular and new types of codes for location-domination. Discrete Appl. Math. 247 (2018), 225-241.

134. M. G. Karpovsky, K. Chakrabarty and L. B. Levitin, On a new class of codes for identifying vertices in graphs, IEEE Trans. Inform. Theory IT-44 (1998), 599-611.

135. Y. Kim, M. Kumbhat, Z. L. Nagy, B. Patkós, A. Pokrovskiy and M. Vizer, Identifying codes and searching with balls in graphs. Discrete Appl. Math. 193 (2015), 39-47.

136. R. Kincaid, A. Oldham and G. Yu, Optimal open-locating-dominating sets in infinite triangular grids. Discrete Appl. Math. 193 (2015), 139-144.

137. T. Laihonen, Sequences of optimal identifying codes. IEEE Trans. Inform. Theory IT-48 (2002), 774776 .

138. T. Laihonen, Optimal codes for strong identification, European J. Combin. 23 (2002), 307-313. 
139. T. Laihonen, Optimal $t$-edge-robust $r$-identifying codes in the king lattice. Graphs Combin. 22 (2006), 487-496.

140. T. Laihonen, Information retrieval and the average number of input clues. Adv. Math. Commun. 11 (2017), 203-223.

141. T. Laihonen and S. Ranto, Families of optimal codes for strong identification. Discrete Appl. Math. 121 (2002), 203-213.

142. A. Lobstein, Watching systems, identifying, locating-dominating and discriminating codes in graphs, a bibliography. https://www.lri.fr/ lobstein/debutBIBidetlocdom.pdf

143. A. McLoughlin, The complexity of computing the covering radius of a code. IEEE Trans. Inform. Theory IT-30 (1984), 800-804.

144. R. Martin and B. Stanton, Lower bounds for identifying codes in some infinite grids. Electron. J. Combin. 17(1) (2010), R122.

145. J. Moncel, Monotonicity of the minimum cardinality of an identifying code in the hypercube. Discrete Appl. Math. 154 (2006), 898-899.

146. B. N. Omamalin, S. R. Canoy, Jr., and H. M. Rara, Differentiating total domination in graphs: revisited. Internat. J. Math. Analysis 8 (2014), 2789-2798.

147. O. Ore, Theory of Graphs, American Mathematical Society, Providence, 1962.

148. A. Parreau, Problèmes d'identification dans les graphes. Thèse de Doctorat, Université de Grenoble, France, (2012), 214 pages.

149. M. Pastori, Les codes identifiants ou comment sauver le Palais des flammes ? Découverte 369 (2010), 56-59.

150. M. Pelto, New bounds for $(r, \leqslant 2)$-identifying codes in the infinite king grid. Cryptogr. Commun. 2 (2010), 41-47.

151. M. Pelto, Maximum difference about the size of optimal identifying codes in graphs differing by one vertex. Discrete Math. Theor. Comput. Sci. 17(1) (2015), 339-356.

152. M. Pelto, On locating-dominating codes in the infinite king grid. Ars Combin. 124 (2016), 353-363.

153. N. J. Rad and H. Rahbani, Bounds on the locating-domination number and differentiating-total domination number in trees. Discuss. Math. Graph Theory 38 (2018), 455-462.

154. H. Rahbani, N. J. Rad and S. M. MirRezaei, Bounds on the identifying codes in trees. Graphs Combin. 35 (2019), 599-609.

155. D. F. Rall and P. J. Slater, On location-domination numbers for certain classes of graphs. Congr. Numer. 45 (1984), 97-106.

156. S. Ranto, Optimal linear identifying codes. IEEE Trans. Inform. Theory IT-49 (2003), 1544-1547.

157. S. Ranto, On binary linear $r$-identifying codes. Des. Codes Cryptogr. 60 (2011), 81-89.

158. N. S. V. Rao, Computational complexity issues in operative diagnosis of graph-based systems. IEEE Trans. Comput. 42 (1993), 447-457.

159. S. Ray, R. Ungrangsi, F. De Pellegrini, A. Trachtenberg and D. Starobinski, Robust location detection in emergency sensor networks. Proceedings of INFOCOM 2003, San Francisco, USA, (2003), 1044-1053.

160. D. L. Roberts and F. S. Roberts, Locating sensors in paths and cycles: the case of 2-identifying codes. European J. Combin. 29 (2008), 72-82.

161. M. L. Roden and P. J. Slater, Liar's domination in graphs. Discrete Math. 309 (2009), 5884-5890.

162. P. Rosendahl, On the identification of vertices using cycles. Electron. J. Combin. 10(1) (2003), R7.

163. S. J. Seo, Open-locating-dominating sets in the infinite king grid. JCMCC 104 (2018), 31-47.

164. S. J. Seo and P. J. Slater, Open neighborhood locating-dominating sets. Australas. J. Combin. 46 (2010), 109-119.

165. S. J. Seo and P. J. Slater, OLD trees with maximum degree three. Util. Math. 94 (2014), 361-380.

166. J. L. Sewell and P. J. Slater, A sharp lower bound for locating-dominating sets in trees. Australas. J. Combin. 60 (2014), 136-149.

167. P. J. Slater, Leaves of trees. Congr. Numer. 14 (1975), 549-559.

168. P. J. Slater, Domination and location in graphs. Research Report No. 93. National University of Singapore, (1983).

169. P. J. Slater, Domination and location in acyclic graphs. Networks 17 (1987), 55-64.

170. P. J. Slater, Dominating and reference sets in a graph. J. Math. Phys. Sci. 22 (1988), 445-455.

171. P. J. Slater, Locating dominating sets and locating-dominating sets. In Graph Theory, Combinatorics, and Applications: Proceedings of the 7th Quadrennial International Conference on the Theory and Applications of Graphs, Wiley, 2 (1995), 1073-1079. 
172. P. J. Slater, Fault-tolerant locating-dominating sets. Discrete Math. 249 (2002), 179-189.

173. P. J. Slater, Liar's domination. Networks 54 (2009), 70-74.

174. B. Stanton, Improved bounds for $r$-identifying codes of the hex grid. SIAM J. Discrete Math. 25 (2011), 159-169.

175. D. P. Sumner, Point determination in graphs. Discrete Math. 5 (1973), 179-187.

176. D. B. Sweigart, J. Presnell and R. Kincaid, An integer program for open locating dominating sets and its results on the hexagon-triangle infinite grid and other graphs. Proceedings of 2014 Systems and Information Engineering Design Symposium (SIEDS), Charlottesville, USA (2014), 29-32.

177. Y. Xiao, C. Hadjicostis and K. Thulasiraman, The $d$-identifying codes problem for vertex identification in graphs: probabilistic analysis and an approximation algorithm. Proceedings of COCOON 2006, 12th Annual International Computing and Combinatorics Conference, Taipei, Taiwan (2006), 284-298.

178. M. Xu, K. Thulasiraman and X. D. Hu, Identifying codes of cycles with odd orders. European J. Combin. 29 (2008), 1717-1720.

179. E. Yaakobi and J. Bruck, On the uncertainty of information retrieval in associative memories. Proceedings of the 2012 IEEE International Symposium on Information Theory (2014), 106-110.

180. J. Yao, X. Yu, G. Wang and C. Xu, Neighbor sum distinguishing total coloring of 2-degenerate graphs. J. Combin. Optim. 34 (2017), 64-70.

181. Z. Zhang, X. Chen, J. Li, B. Yao, X. Lu and J. Wang, On adjacent-vertex-distinguishing total coloring of graphs. Sci. China, Ser. A 48 (2005), 289-299. 